\title{
Biological complexity facilitates tuning of the neuronal parameter space
}

${ }^{*}$ Marius Schneider ${ }^{\mathrm{a}, \mathrm{b}, \mathrm{c}, \mathrm{d}}$, Albert Gidon ${ }^{\mathrm{e}}$, Jochen Triesch ${ }^{\mathrm{a}, \mathrm{d}, \mathrm{f}}$, Peter Jedlicka ${ }^{\mathrm{a}, \mathrm{c}, \mathrm{g}, \mathrm{l}, 1},{ }^{*}$ Hermann Cuntz $^{\mathrm{a}, \mathrm{b}, 1}$

${ }^{a}$ Frankfurt Institute for Advanced Studies, 60438 Frankfurt am Main, Germany

${ }^{b}$ Ernst Strüngmann Institute (ESI) for Neuroscience in cooperation with the Max Planck Society, 60528 Frankfurt am Main, Germany

${ }^{c}$ ICAR3R - Interdisciplinary Centre for 3Rs in Animal Research, Justus Liebig University

Giessen, 35390 Giessen, Germany

${ }^{d}$ Faculty of Physics, Goethe University, Frankfurt/Main, 60438 Frankfurt am Main, Germany ${ }^{e}$ Institute for Biology, Humboldt-Universität zu Berlin, Berlin, Germany

${ }^{f}$ Faculty of Computer Science and Mathematics, Goethe University, 60438 Frankfurt am Main, Germany

${ }^{g}$ Institute of Clinical Neuroanatomy, Neuroscience Center, Goethe University, 60528 Frankfurt am Main, Germany

${ }^{1}$ Joint senior authors

*mschneider@fias.uni-frankfurt.de, cuntz@fias.uni-frankfurt.de

\section{Keywords}

Dendrite function, Degeneracy, Electrophysiology, Compartmental modelling, Evolutionary principles, Law of large numbers 


\section{In brief}

Studying ion channel diversity in neuronal models we show how robust biological systems may evolve not despite but through their complexity.

\section{Highlights}

- 15 channel model of hippocampal granule cells (GCs) reduces to 5 ion channels without loss of spiking behaviour.

- But knocking out ion channels can be compensated only in the full model.

- Random sampling leads to $\sim 6 \%$ solutions in full but only $\sim 1 \%$ in reduced model.

- Law of large numbers generalises our observations to other complex biological systems. 


\section{Abstract}

The electrical and computational properties of neurons in our brains are determined by a rich repertoire of membrane-bound ion channels and elaborate dendritic trees. However, the precise reason for this inherent complexity remains unknown. Here, we generated large stochastic populations of biophysically realistic hippocampal granule cell models comparing those with all 15 ion channels to their reduced but functional counterparts containing only 5 ion channels. Strikingly, valid parameter combinations in the full models were more frequent and more stable in the face of perturbations. Scaling up the numbers of ion channels artificially in the reduced models recovered these advantages confirming the key contribution of the actual number of ion channels. We conclude that the diversity of ion channels allows a neuron to achieve a target excitability through random channel expression with increased robustness and higher flexibility.

\section{Significance statement}

Over the course of billions of years, evolution has led to a wide variety of biological sys- 14 tems. The emergence of the more complex among these seems surprising in the light of the high demands on searching viable solutions in a correspondingly high-dimensional parameter space. In realistic neuron models with their inherently complex ion channel composition, we find a surprisingly large number of viable solutions when selecting parameters randomly. This effect is strongly reduced in models with less ion channel types but is recovered when inserting additional artificial ion channels. Because concepts from probability theory provide a plausible explanation for such an improved arrangement of valid model parameters, we propose that this generalises to evolutionary selection in other complex biological systems. 


\section{Introduction}

Throughout evolution, biological cells have emerged with increasing diversity and complexity. Optimising for multiple objectives while keeping an ever larger number of cell parameters within a viable range seems a daunting task for evolutionary processes. And it remains unclear how such a multi-objective optimisation can be achieved in the corresponding high dimensional parameter space. Here we explore the counter-intuitive hypothesis that increasing the number of mechanisms - i.e. increasing the biological complexity - potentially helps to evolve more quickly, easily and efficiently towards satisfying a large number of objectives.

Neurons are a good example for complex cells typically exhibiting a great diversity in the expression of ion channels as products of such evolutionary optimisation. The channel parameters must be tuned to cooperatively generate multiple features of neuronal spiking behaviour. A palette of such spiking features has been successfully used in computational biophysical neuron models for multi-objective optimisation (MOO) using genetic algorithms (Druckmann, 2007). Mammalian neurons contain a large variety of ion channels in their membrane (Coetzee et al., 2006) producing a wide range of possible spiking mechanisms with varying temporal dynamics and excitability (Connors and Gutnick, 1990). Interestingly, a number of these ion channel variants exhibit overlapping functional properties (Coetzee et al., 2006; Rudy, 1988; Herrera-Valdez et al., 2013; Marder and Goaillard, 2006; Olypher and Calabrese, 2007; Hille, 2001). A large body of literature has explored the reason for this high diversity (Marder, 2011; Prinz et al., 2004; Golowasch et al., 2002; O'Leary et al., 2013). However, it remains unclear what role exactly does the diversity of ion channels play regarding evolution and its contribution to functional mechanisms that impact neuronal computations.

Neuronal computation relies on the morphology as well as on the diversity and distribution of ion channels in the membrane of the dendritic tree, the soma and the axon initial segment. Even small changes in the distribution of ion channels can change the activity in neurons drastically (Achard and De Schutter, 2006). Large differences in experimental measurements have been observed from cell to cell, day to day and animal to animal in data from the same classes of cells (Marder and Goaillard, 2006; Golowasch et al., 2002; Golowasch and Marder, 1992; MacLean et al., 2003; Swensen, 2005; Schulz et al., 2006, 2007). The expression 
and Goaillard, 2006; Prinz et al., 2004; Golowasch et al., 2002; Golowasch and Marder, 1992; MacLean et al., 2003; Schulz et al., 2006). However, many detailed biophysical models of single cells ignore this variability in electrophysiological data and search for a fixed set of parameters that replicates an average behaviour of a particular cell type (Golowasch et al., 2002).

One reason for this ion channel diversity might be the fact that gene expression is a rather stochastic process (Raj and van Oudenaarden, 2008). It is known that gene expression is subject to random fluctuations leading to cell-to-cell variations in mRNA and protein levels (Elowitz et al., 2002). How can neurons then still manage to achieve a functional target activity? Using a spike-feature-based multi-objective approach, we generated large population parameter sets of dentate granule cell (GC) models with different numbers of ion channel types in order to investigate the potential advantages of ion channel diversity. We then tested to which degree the different models could compensate for pathological channel loss. Furthermore, we investigated differences in functional parameter sets, taking into account stochastic fluctuations in channel-coding gene expression. Finally, we studied the stability of the different models against ion channel alterations due to e.g. protein turnover. We found that in all cases the complete GC model with all ion channels was more robust, stable and had more valid parameter combinations than its reduced counterparts.

\section{Results}

We used a recently established multi-compartmental model comprising the 15 different voltage 74 or calcium-dependent ion channels that were described in mouse GCs (Beining et al., 2017). The model was specifically designed to reproduce the results not of a single experiment but of a large series of experiments and was based on raw electrophysiology traces. Its parameters were fitted to reproduce the experimental data for a number of different reconstructed (see example in Figure 1A, Top, from Schmidt-Hieber et al., 2007) and synthetic neuronal morphologies making the model robust within the GC morphological space. Furthermore, the resulting model readily generalised to rat GCs as well as to adult born mouse GCs after incorporating the known changes in morphology and ion channel composition. The model can therefore be considered to be robust and comprehensive. This makes it an experimentally validated tool to study the impact of complex ion channel compositions on robustness of the spiking 
output. To this end, we employed a population-based (also called "ensemble" or "database") 85 modelling approach, which allowed us to explore the multidimensional parameter space in 86 large populations of stochastically generated models (Prinz et al., 2003; Gunay et al., 2008; 87 Britton et al., 2013; Sekulic et al., 2014; Rathour and Narayanan, 2019).

\section{The GC model cost function}

First, we developed a cost function for an automated evaluation of the validity of diverse models, which differed in their ion channel combinations and densities. Since no quantitative data exists on the particular expression of the various ion channels in individual GCs, some form of fitting procedure of channel densities was required in the construction of the GC model. The model consists of 27 conductance parameters, which precludes a grid scan for parameter fitting due to the long computing time in a 27 dimensional parameter space. The 94 model has therefore previously been largely tuned manually with expert knowledge from GC biology. To assess the quality of any individual set of parameters more automatically, we designed a fitness function that quantified the distance to experimental spiking data and was inspired by approaches used previously (Druckmann, 2007; Beining et al., 2017, see Methods, Figure S1. A number of different methods have been proposed to quantify the quality of a set of parameters in relation to neuronal activity (Achard and De Schutter, 2006; Bahl et al. 2012; Keren et al., 2005; Vanier and Bower, 1999). While most studies focus on reproducing an average electrophysiological activity pattern, we wanted to the distribution of valid parameter combinations in the GC model taking into account the variability present in experimental data. 
A

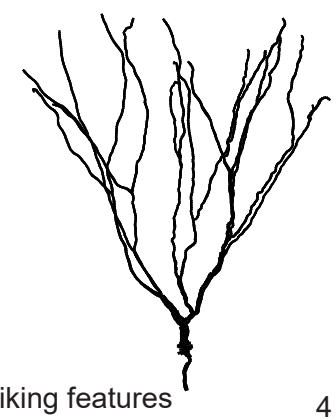

Spiking features

1. Firing rate

2. Spike latency

3. Voltage threshold

4. Spike amplitude

5. FAHP

6. Absolute fAHP

7. Spike width

8. First ISI

9. Spike freq. adaptation

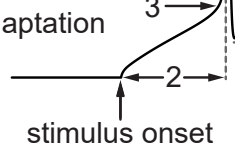

C

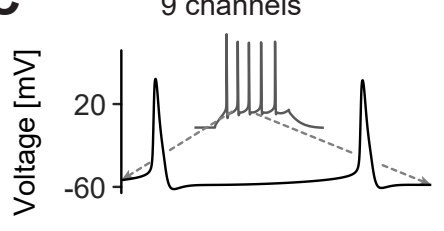

$\frac{\Xi}{+}$

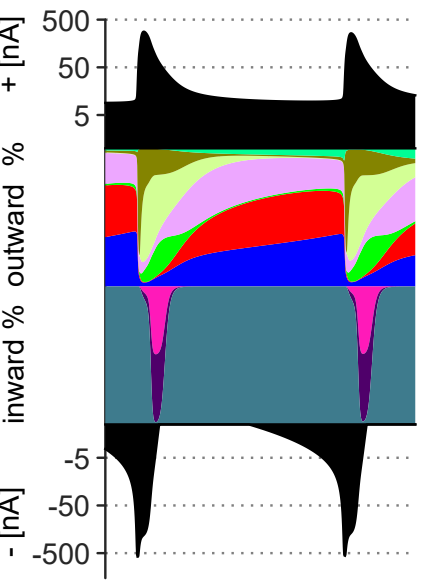

$\overline{50 \mu \mathrm{m}}$
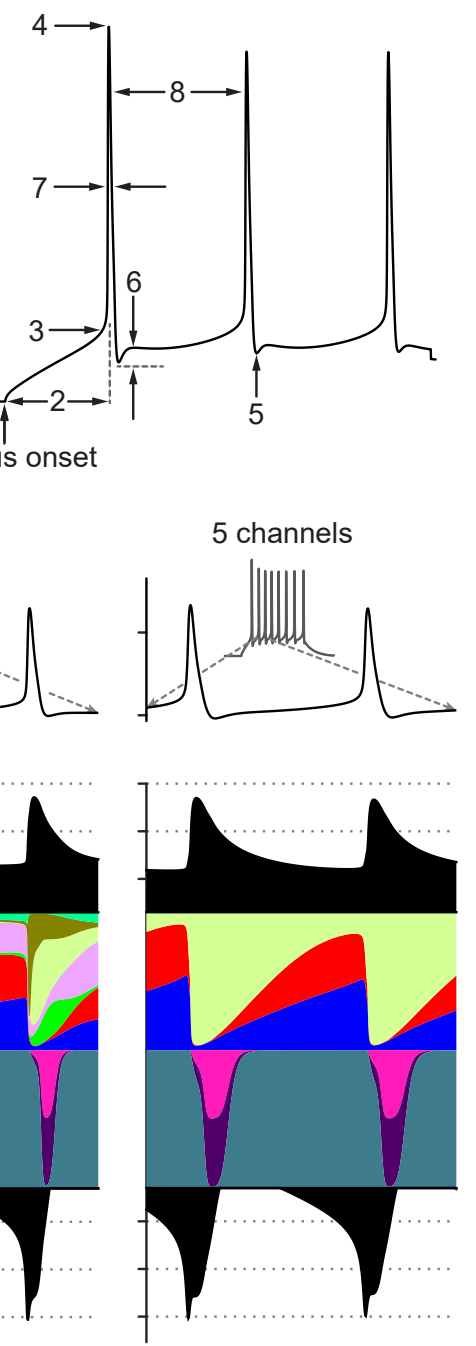

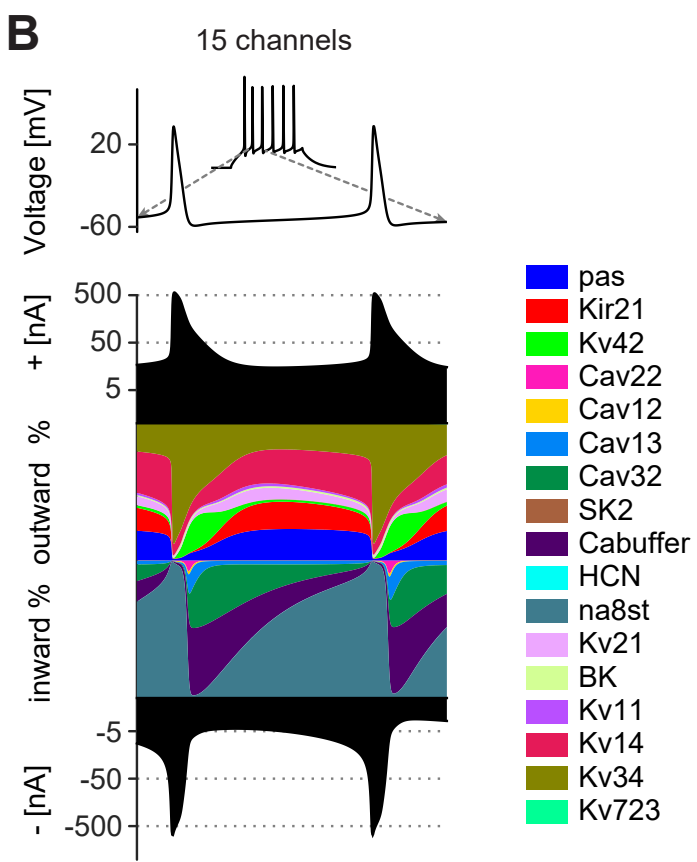

15 channels - BK

15 channels - Cav22
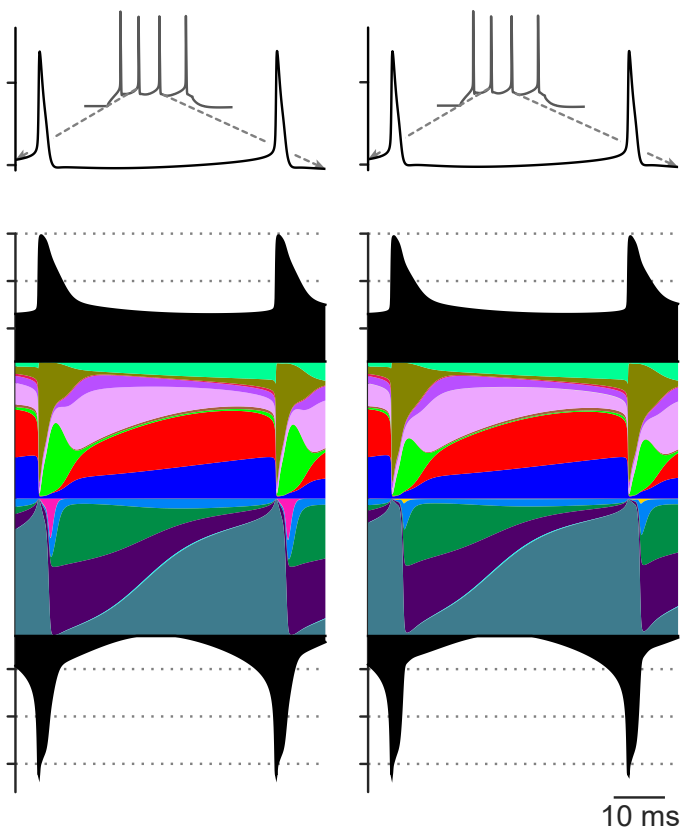

Figure 1. Simplified as well as realistic complex ion conductance-based models capture multiple spiking features of real granule cells (GCs)

A, (Top) 3D-reconstructed mouse GC morphology used for our simulations (Schmidt-Hieber et al. 2007). (Bottom) Spike features used to calculate the multi-objective fitness of the GC model. B, Membrane potential during $200 \mathrm{~ms}$ lasting current clamp of $90 \mathrm{pA}$. The coloured curves show the relative contribution of all implemented ion channels to the total inward and outward current at each time step (during the second and third spike) as a percentage of the total current. The black filled curves illustrate the total inward and outward currents on a logarithmic scale. This plot was inspired by Alonso and Marder (2019). See next page. 
Figure 1. (continued) C, Contribution of currents to total inward and outward current in reduced models and models that compensate for the knock out of the BK (Left) and Cav22 (Right) channel. Similar visualisation and current injection procedure as in $\mathbf{B}$.

We therefore used a multi-objective fitness function based on spike features, which allowed us to search for optimal trade-offs between different firing properties (Druckmann, 2007). We extracted 9 different spiking features from raw electrophysiology traces during a $200 \mathrm{~ms}$ current clamp injection with 50 and $90 \mathrm{pA}$ at the soma (Figure $1 \mathrm{~A}$, Bottom, see Methods). We then compared the values for these features between the model and the experimental data. To generate a population of GC model instances that reflected the full range of firing properties, we calculated the deviation from the experimental mean in units of experimental standard deviation (SD) (Druckmann, 2007). In order to become a valid parameter combination in the GC model, the error value was required to be less than two SDs away from the experimental average of each feature.

A manual search for parameter sets fulfilling this requirement was very time-consuming 116 and could never be exhaustive. There are various automated parameter search methods, 117 such as gradient descent methods, genetic algorithms, simulated annealing and stochastic ${ }_{118}$ search methods, which make the search for parameters more efficient (Vanier and Bower. 1999; ${ }_{119}$ Mitchell, 1998; Kirkpatrick et al., 1983: Press et al., 2007). Since we were starting from a valid 120 parameter combination, we decided to use a gradient descent algorithm (Press et al., 2007) in 121 combination with random parameter space exploration (see Methods). This method led to 122 good parameter combinations within a few iteration steps also when starting from random ${ }_{123}$ parameter sets for which the model deviated from the experimental results. The gradient ${ }^{124}$ descent method was even capable of finding parameter combinations when starting from 125 initial parameter sets for which the models produced no spikes at all (see details in Figure S2). ${ }_{226}$

\section{Reduction of channel diversity}

Electrophysiological signatures of neurons of a same class are often unique allowing a loose classification of cell types by their electrophysiology. However, the spiking mechanisms often include multiple ion channels with overlapping functionality to achieve these specific spiking behaviours (Coetzee et al., 2006: Olypher and Calabrese, 2007; Marder, 2011; Bean, 2007; O'Leary et al. 2014; Drion et al., 2015). Thus, an important question is, how many channels are 
functionally necessary for a given cell type. We addressed this question in GCs, which have a relatively simple electrophysiological repertoire, but, surprisingly, their membrane contains a large palette of voltage- and calcium-dependent conductances (Beining et al., 2017). The compact activity together with the multitude of ion channels in the corresponding GC model (Figure 1C) suggests that a reduction of channels without losing accurate model performance might be possible. Therefore, we explored this possibility by incremental simplification of the GC model. First, we reduced the number of voltage-dependent conductances in the highly detailed multi-compartmental model of GCs by 6 channels (removing Cav12, Cav13, Cav32, Kv11, Kv14, SK, Figure 1C, Leftmost). Thereupon, we gradually reduced the number of remaining channels to a minimum of 5 ion channels (leaving only the leak channels pas, as well as Kir21, Na8st, BK and Cav22) finding parameter combinations that satisfied our cost function using the search algorithm (Figure 1C, Center left).

To visualise the contribution of individual currents to neuronal model activity, we employed a recently developed method of plotting the time course of the relative contribution of each ionic current (Alonso and Marder, 2019). Overall, as expected, the electrophysiological activity of the different valid models in Figure 1C was similar (for overview, see Figure S3). Despite the large variations in the number of ion channels, the course of the total inward and outward current flow displayed only slight changes between the three different baseline models (Figure 1B, C). Since GCs had a simple electrophysiological repertoire, a small number of membrane time constants was sufficient to generate adequate firing patterns. The presence of $\mathrm{K}^{+}$and $\mathrm{Ca}^{2+}$ channels with overlapping physiological functionality ensured that many of the channels were not crucial for the maintenance of functional activity. Only the composition of the inward and outward currents differed. In the 5-channel model, the calcium-sensitive potassium channel (BK) took over the role that 8 different $\mathrm{K}^{+}$conductances had shared in the non-reduced model (Figure 1C). BK thereby became the only remaining $\mathrm{K}^{+}$channel overall. In interaction with the $\mathrm{Ca}^{2+}$ conductances (Cav22), the BK channel was responsible for repolarising the membrane potential following an action potential in the 5 -channel model.

Recent experimental and theoretical studies demonstrated that neurons can compensate for 
model where less redundancy exists. Indeed, we found that blocking the BK or N-type 165 Cav22 channels in the full model was readily rescued by contributions from other channels 166 (Figures 1C, Right). It is noticeable that the loss of the BK channel was compensated by a 167 strong upregulation of another calcium-sensitive channel (SK), as well as of voltage-dependent 168 potassium channels (Kv 7.2/3, Kv 1.1, Kv 2.1, Figure S5, Left). The fact that blocking the SK channel under normal conditions (unreduced model) had only a minor effect on the behaviour of the GC model suggests that the SK channel acts as a backup (but see also Mateos-Aparicio et al., 2014). Neither loss of BK nor Cav22 could be compensated for in the reduced 5-channel model since it had only one active gating mechanism per ion type. Even the 9 -channel model was not able to compensate for the pathological loss of Cav22 or BK. As expected, therefore, the full GC model's diversity contributed to the model's robustness with respect to the loss of specific ion channels through existing ion channel redundancies.

\section{9}

\section{Random parameter tuning as a viable approach to selecting GC model}

Even though small changes in the ion channel expression level can already lead to drastic changes in neuronal activity, several experimental studies observed that intrinsic properties of nerve cells can vary considerably across neurons of the same type (Golowasch et al., 2002; Golowasch and Marder, 1992; MacLean et al., 2003; Swensen, 2005; Schulz et al., 2006, 2007). Moreover, theoretical investigations demonstrated that indistinguishable network and single neuron activity can be obtained from a large variety of model parameter settings (Prinz et al. 2004: Golowasch et al. 2002). This raises the question of whether the diversity of voltage- and calcium-dependent conductances has an effect on the variability of valid parameter sets in the GC model leading to realistic spiking activity.

In order to check this, we first generated 20,000 random model instances for each of the three baseline models by randomly sampling the individual conductance densities within a range between $0 \times$ and $2 \times$ the value in the baseline model. As the ohmic relations between current and voltage were consistent with experimental results in all cases (see Figure $\mathbf{S 3} \mathbf{B}$ ), we did not change the densities of the leak channel or the inward-rectifying Kir21 channel, which primarily contribute to the passive properties of the neuron. The population of functional parameter combinations enabled us to calculate the Pearson's correlation coefficient $r$ for all pairs of conductance density parameters. We found weak mutual correlations indicating low 
dependencies between the channels thus increasing the robustness of the model (Figure S4). 195 The strongest correlation was observed between the expression levels of the $\mathrm{Na}^{+}$channel in 196 the soma and in the AIS ( $r=0.91)$. The sodium channel is essential for spike initiation and its 197 presence in different regions of the GC suggests that compensatory mechanisms could simply 198 be instantiated by maintaining a balance between the same currents in different regions, which 199 results in a significant anticorrelation. Interestingly, the reduced models showed stronger and different correlations between the channels than the full model.

In our selection of random parameter combinations, we found suitable models covering the entire sample range of the majority of parameters (Figure 2A). In all cases, the most constrained parameter was the density of the 8 -state $\mathrm{Na}^{+}$channel. Since the $\mathrm{Na}^{+}$channels were implemented as a Markov chain model with one common maximum conductance parameter shared by all of its states, it is not surprising that they were not as variable as the other conductances. In addition, the reduction of channel diversity in the 5-channel model limited the variability of the calcium-dependent potassium channel BK (Figure 2A, Right). ${ }_{208}$ Surprisingly, the overall percentage of randomly selected parameter combinations that were 209 valid increased with the number of ion channels (Figures $\mathbf{2} \mathbf{B}, \mathbf{C}, \sim 0.7 \%$ with 5 channels, 210 $\sim 3.3 \%$ with 9 channels, and $\sim 5.7 \%$ with 15 channels).

The distribution of voltage- and calcium-activated channels in cell membranes is under 212 continuous regulation (Raj and van Oudenaarden, 2008; Gal et al., 2010; Marder et al., 2014). 213 On the one hand, the cell is subject to homeostatic regulation maintaining its electrical activity 214 despite changes in its environment and input. On the other hand, the proteins are constantly 215 exchanged during the lifetime of a cell. In order to investigate the stability of the valid 216 parameter combinations in the different models in face of parameter perturbations due to e.g. 217 protein exchange during the lifetime of a cell, we performed random walks in the parameter 218 space. Starting from a valid parameter set that accurately reproduced the experimentally 219 derived behaviour, we iteratively changed each parameter by random steps between $-5 \% \quad 220$ and $+5 \%$ of the current parameter values (counting changes in all parameters as one step). 221 The random walk stopped as soon as the parameter combination became invalid, i.e. the 222 cost function for the resulting model increased beyond 2 standard deviations away from 223 experimental results. Interestingly, the average number of possible random parameter changes 224 before model failure increased with the number of ion channels in the models (Figure 2D). 225 
A
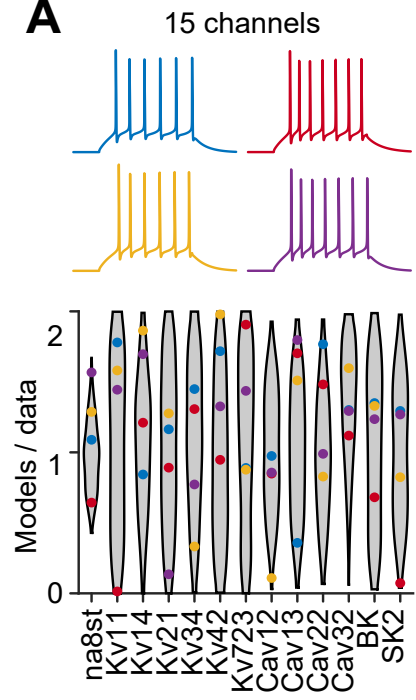

B

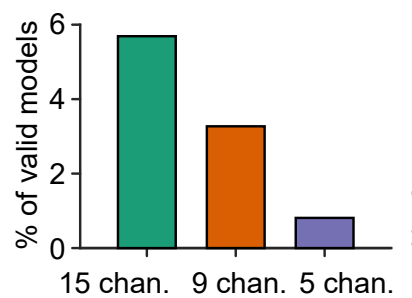

15 chan. 9 chan. 5 chan.

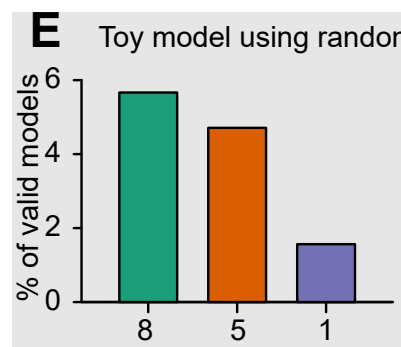

Number of variables
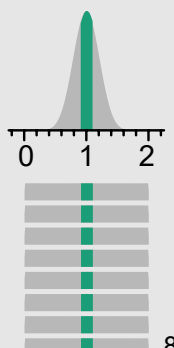
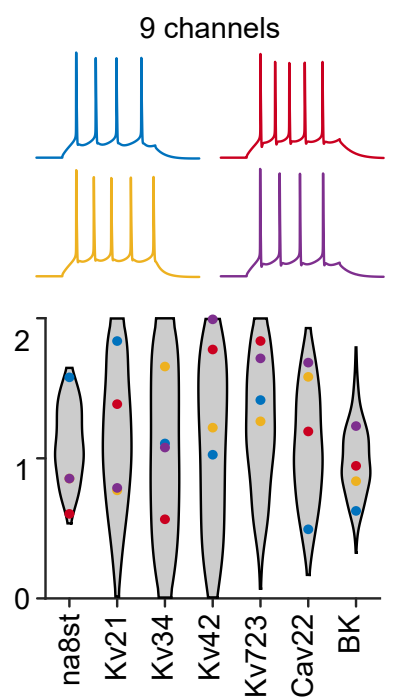

C

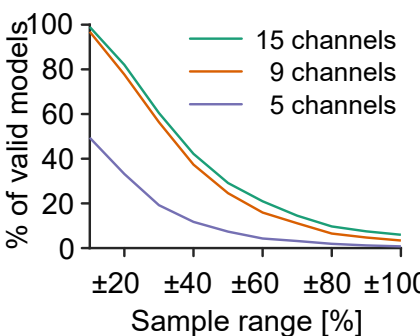

Sample range [\%]

variables
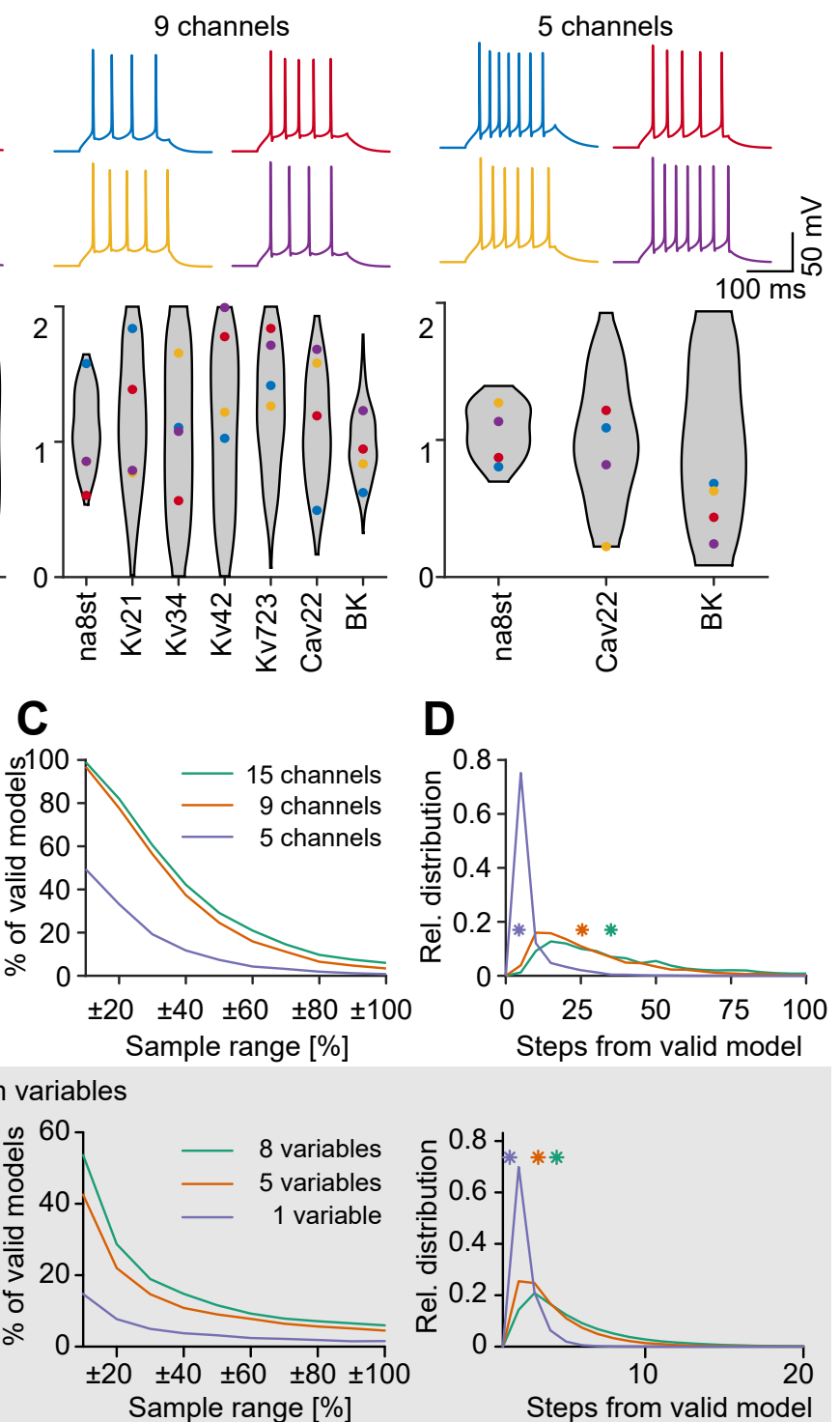

Figure 2. Valid parameter combinations in the fully complex model are well spread and more stable as compared to reduced models.

A, (Top) Activity traces of 4 randomly picked valid parameter combinations in each of the GC models of different complexity. (Bottom) Coloured dots illustrate conductance densities of the four valid parameter combinations shown in top traces. The grey violin plots delimit the entire range covered by the valid parameter combinations. Conductances are weighted by the surface area of the corresponding membrane regions. 
Figure 2. (continued) B, Percentage of valid random parameter combinations in the total population in the 2 -fold range. $\mathbf{C}$, Percentage of valid random parameter combinations in samples with different ranges around the valid reference parameter combination. Each sample contained 5,000 parameter combinations. D, Random walk through the parameter space starting from valid combinations in models of different complexity. Relative percentage distribution of the maximum number of random steps the respective models could undergo without losing their valid GC spiking behaviour. Bin size is 4 steps. Asterisks indicate mean number of steps the corresponding models could undergo while maintaining realistic activity. Performed for 2, 000 repetitions per each model. B-D, Colours were Green: full model, Orange: 9 -channel model, Purple: 5 -channel model. E, Reproduction of B-D with a toy model representing the model result as the average value of 1 (blue), 5 (red) and 8 (green) uniform random variables between 0 and 2. Bottom panels, Illustration of how the distribution of solutions becomes narrower when the number of variables is increased. This effect is explained by the law of large numbers while the Gaussian distributions results from the central limit theorem.

\section{Toy model points to law of large numbers}

As shown in the previous sections, we observed an increase in valid random parameter sets when biophysical models of neurons became more complex. One possible explanation could be the fact that the more complex models included different ion channels of the same type. Since some of these ion channels show very similar gating dynamics (see for example Cav22, Ca12 and Cav13, see Figure 1) their functional contributions may be partially redundant. A theorem from probability theory, namely the law of large numbers can play a role under such circumstances. The law of large numbers states that increasing the number of samples (in our case ion channels of a similar type) described by a random variable will move the average over the samples closer to the expected mean value. In other words, for example, throwing dice between 1 and 6 more often will lead to a weighted average that is closer to the expected value 3.5. Since in our case we sample conductances of similar ion channels, the average conductance would therefore converge towards the starting parameter set that we know is functional.

In order to illustrate this we designed a simple toy model using random variables for each parameter. Here, we represented each open parameter of the model by one random variable with a homogeneous probability of throwing any number between 0 and 2 corresponding to the parameter ranges used in the neuronal model between $0 \times$ and $2 \times$ the default value (Figure 2, Bottommost). To keep things really simple, we set the model outcome to be the statistical mean of the values of all separate random variables. The law of large numbers predicts a decreasing variance around the mean value with increasing number of random 
variables as illustrated in the sketch at the bottom of Figure $2 \mathbf{E}$. The central limit theorem 247 in turn predicts a Gaussian distribution regardless of the probability distributions for each 248 random variable separately. In analogy to our neuronal modelling, we then constrained valid 249 parameter combinations by a cost function allowing a maximal distance of here 0.015 away 250 from the mean value, i.e. 1, averaged over all random variables.

The analogy here is limited since in contrast to the channels in the GC model all variables in 252 our toy model are functionally the same. Moreover, the GC compartmental model applies 253 complex nonlinear and dynamic transformations of the starting parameter space including 254 distinct jumps in the cost function for example when the model no longer produces action 255 potentials. However, despite its simplicity, our toy model was able to reproduce all results from our GC model in Figure 2B-D qualitatively (Figure 2E). The law of large numbers therefore provides a plausible explanation why a larger number of random instances in the more complex neuron model would more readily linger around their target functionality.

\section{Additional model robustness through artificial ion channel isoforms}

We have shown that the electrophysiological behaviour of GCs can be maintained despite a reduction of ion channel diversity from 15 channels to 5 channels. However, our results also suggest that this loss of ion channels goes along with a decrease in stability, a loss of compensatory opportunities and a significant decrease in the valid model percentage within a randomised sample. From our toy model based on simple probability theory we postulate that it might be the mere number of ion channels that contribute to the increased robustness observed in the full model rather than the particular ion channel composition present there. In order to validate this hypothesis, we chose to start from the reduced model and increase the number of ion channels in an artificial way to check whether we could recover the robustness present in the realistic full model.

In order to establish a quantitative relation between channel diversity and model stability in such a way, we scaled up the 5 -channel model's diversity by adding more instances of the calcium (Cav22) and potassium channels (BK) remaining in that model. These artificial isoforms of the existing ion channels distinguished themselves from the original Cav22 and BK by randomised time constants (within a two-fold range of the original parameters) to allow for different dynamics through the new ion channel isoforms. 

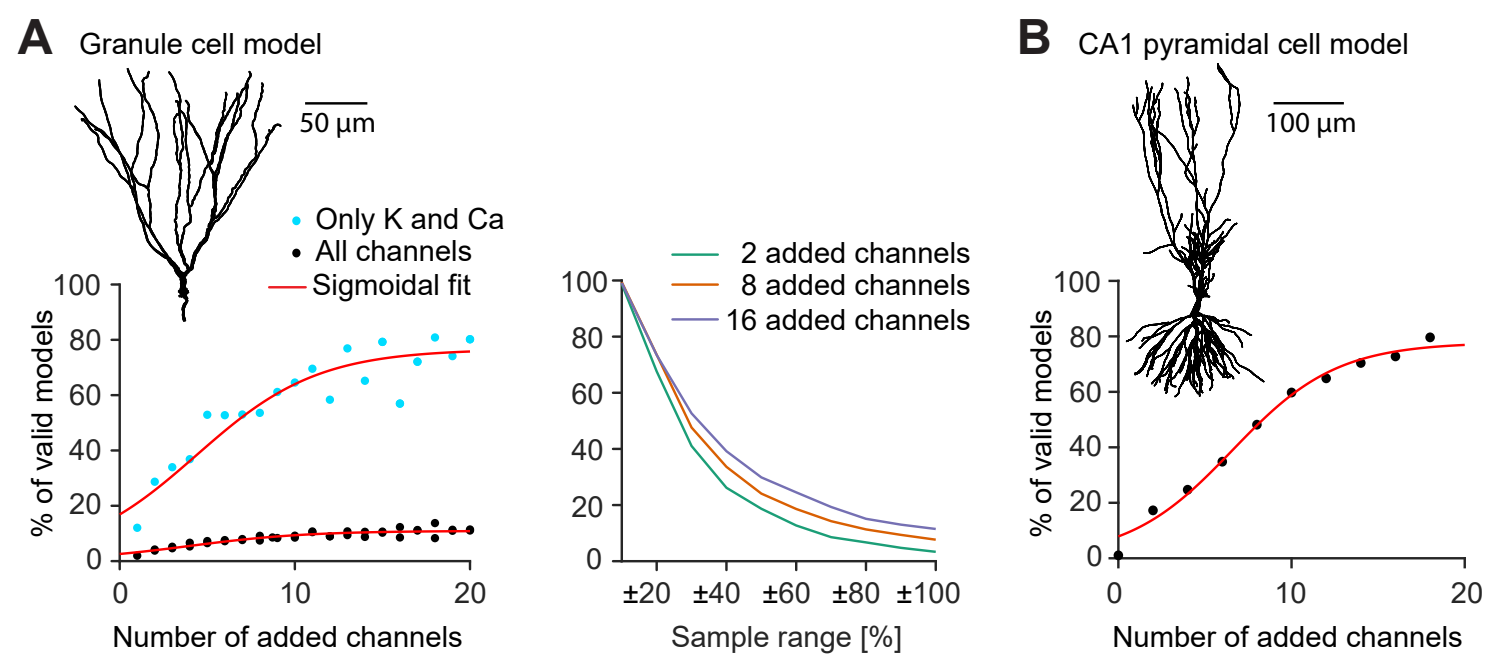

Figure 3. Artificial ion channel diversity expansion recovers and enhances the proportion of valid parameter combinations in the reduced 5-channel model

A, Populations of expanded dentate GC models with $0-35$ added artificial ion channel isoforms. Left panel, The plot shows the percentage of functional parameter combinations in a population of randomly sampled channel densities. Black dots show the populations where all ion channels (including the 8 -state Markov chain modelled $\mathrm{Na}^{+}$channel) were sampled in a $0-2 \times$ range. Blue dots show the populations where only potassium and calcium channels were sampled in a $0-2 \times$ range. Right panel, Similar plot as in Figure 2C for the black models from the left panel. B, Similar overall analysis as in A but for a CA1 pyramidal cell model (Jarsky et al., 2005).

To examine the proportion of valid parameter combinations with increasing number of ion ${ }_{277}$ channels, we created a multitude of functional GC models with up to 35 additional ion channel 278 isoforms. For each given number of ion channel isoforms, we randomly sampled all conduc- 279 tance values in a two fold range. Thereupon we selected the three parameter combinations 280 with the best fitness value for each number of ion channel isoforms and improved their ${ }^{281}$ performance by applying a gradient descent algorithm. We then followed the same procedure ${ }_{282}$ as in Figure 2. Using this approach, the percentage of valid parameter combinations steadily ${ }_{283}$ increased with the number of additional ion channel isoforms until reaching a plateau between ${ }^{284}$ 15 and 20 additional ion channel isoforms (Figure $3 \mathbf{A}$ ). To further generalise our findings 285 in Figure $3 \mathrm{~A}$ we have applied the same procedure to a different neuronal model type, one 286 simulating a CA1 pyramidal neuron (Jarsky et al. 2005: Cuntz et al. 2019, Figure 3B). Viewed ${ }_{287}$ together, these results show the major contribution of ion channel diversity by demonstrating 288 that scaling up the numbers of ion channels artificially in the reduced models leads to more ${ }_{289}$ frequent valid parameter combinations. This is in line with the law of large numbers. 


\section{Discussion}

In this study, we explored the complex landscape of valid parameter combinations in a 292 parameter space of a detailed multi-compartmental model of dentate GCs and its simplified versions with reduced ion channel complexity (Figure 1). We used a population-based approach (Gunay et al., 2008; Marder, 2011; Britton et al., 2013; Sekulic et al., 2014), in order to find multiple ion channel parameter combinations for models that successfully reproduced the electrophysiological data (Figures 2 and S1). We show that the biologically realistic GC model (full model) with many redundant ion channel types was more robust to ion channel perturbations than valid models with reduced ion channel diversity. Importantly, noisy ion channel expression simulated by random parameter combinations produced $\sim 6 \times$ more valid GC model instances in the full model as compared to the reduced models (Figure 2). The robustness in the reduced model was recovered when adding artificial isoforms of existing ion channels (Figure 3) indicating that it is indeed the number of channels that produces this effect. We argue that this increased robustness comes in part from a direct consequence of basic probability theory.

\section{Robustness through ion channel redundancy in complex GC models}

Most neurons contain more than a dozen different ion channels. While early computational models implemented considerably fewer channels than known in biology, more and more models exist that contain a realistic number of mechanisms (e.g. Beining et al., 2017; Hay et al. 2011). Although the different potassium channels in mammalian cortical neurons differ genetically, some are remarkably similar in their functional contribution to the electrophysiological activity of neurons (Coetzee et al., 2006; Drion et al., 2015). This functional similarity is often referred to as degeneracy (Goaillard and Marder, 2021) and is not a phenomenon restricted to neurobiology (Edelman and Gally, 2001; Tononi et al., 2002). Depending on the computations a neuron should implement, its dynamics only need to cover certain relevant time scales, e.g. 315 in the form of different time constants of its gating variables (Gjorgjieva et al., 2016). Since 316 five channels were sufficient to support realistic voltage dynamics at relevant time scales, we 317 were able to reduce the original variety of ion channels without observing a significant loss 318 in the performance of the model. In our study, GCs with their compact electrophysiological 319 
repertoire did not require a large variety of ion channels to reproduce their characteristic 320 activity patterns. To replicate the 9 experimentally derived spiking properties, the models 321 required only one active channel of each of the different subgroups of ion channels (one $\mathrm{Na}^{+}-,{ }^{3} 2$ one $\mathrm{K}^{+}$- and one $\mathrm{Ca}^{2+}$-channel, as well as the leak channels; Figure $\mathbf{1} \mathrm{C}$ ).

Experimental as well as theoretical studies from the last decades revealed that pharmaco- ${ }^{324}$ logical manipulations like the blockage or upregulation of intrinsic or synaptic mechanisms, 325 resulting in a pathological cellular activity on a short timescale, can be compensated by up- 326 and downregulation of the remaining conductances on a long timescale (MacLean et al., 327 2003: Swensen, 2005; O'Leary et al., 2014; Drion et al., 2015; Guo et al., 2005; Nerbonne et 328 al., 2008; Stegen et al., 2012; MacLean et al., 2005). Interestingly, not all manipulations can 329 be compensated by mechanisms of homeostatic regulation (Zhang et al., 2003), indicating differences in the capability of homeostatic compensation between ion channels as well as types of neurons. As opposed to other studies using biophysically realistic mechanisms of homeostatic intrinsic plasticity based on calcium signals ( $\mathrm{O}^{\prime}$ Leary et al., 2013, 2014; Abbott and LeMasson, 1993: Golowasch et al., 1999; Liu et al., 1998), we decided to use a gradient descent approach to investigate the large and complex parameter space of possible intrinsic compensations. We chose this mathematical approach also because the biophysical mechanisms of intrinsic plasticity are not yet fully understood in detail. The implementation of biophysically incomplete mechanisms of intrinsic plasticity could lead to unnecessary limitations on the regulatory mechanisms.

We demonstrated that the full GC model was capable of compensating the loss of any 340 potassium and calcium channels by up and down regulation of the remaining ion channels (Figure 1C). In contrast, the different reduced models relied on the presence of certain indispensable ion channels, without which they could not capture main electrophysiological characteristics of GCs. Figure $\mathbf{S 5}$ shows that there can be as much as a 20-fold variability in the density of voltage-dependent ion channels. Experimental studies have observed variations of a similar order of magnitude as a result of compensatory mechanisms (MacLean et al., 2003). The ability of these models to compensate for losses of ion channels can be attributed to the overlapping or degenerate physiological function of the present potassium and calcium channels (Mishra and Narayanan, 2021).

The reduction of the diversity of gating mechanisms goes along with a loss of space to 
al. 2015). A strong indication for this conclusion is for example the existence of the calcium- 352 sensitive voltage-dependent potassium channels BK and SK. In the 5 channel model, the ${ }_{353}$ former is of great importance and can significantly disturb the dynamics of the GC model, 354 even with rather small changes in maximum conductance, whereas the SK channel is absent. 355 In case of a loss of the BK channel, the expression level of the SK channel, together with that 356 of other channels, increased strongly and thus maintained the functional behaviour of the ${ }^{357}$ cell. In line with the concept of degeneracy (Druckmann, 2007; Aizenman et al., 2003), the 358 overlapping functionality of different channels enabled the neuron, depending on the given conditions, to achieve a target spiking behaviour in a number of different ways.

In addition, we tested the stability of the differently reduced models against random parameter 361 perturbations, in order to simulate putative protein exchange during the lifetime of a cell. The 362 ongoing protein replacement is one of the reasons for the continuous regulation of voltage- ${ }_{363}$ and calcium-dependent channels in cell membranes (Raj and van Oudenaarden, 2008; O'Leary ${ }_{364}$ et al., 2014: Gal et al. 2010). Although no homeostatic tuning mechanism with a dynamic 365 feedback was implemented, valid parameter combinations in the complete model were able to endure far more random parameter perturbations while maintaining realistic activity than the ones in the reduced models (Figure 2D). This is in agreement with experimental studies, which have shown that, although homeostatic tuning rules can compensate for many perturbations and knock-outs of ion channels, not all channel deletions and perturbations can be compensated (Zhang et al., 2003). A challenge for future experimental work will be to uncover the long-term effects of ion channel knock-outs in GCs in order to find out whether our theoretical results of the outstanding robustness of GCs against channel deletions can be observed in biology.

\section{Random parameter selection as a viable fitting strategy for neurons}

Like many biological processes, gene expression is an essentially stochastic process resulting in heterogeneity of mRNA and protein levels (Raj and van Oudenaarden, 2008: Gal et al., 2010; Sigal et al., 2006). This noise in gene expression is one reason for the cell-to-cell variability. However, noise in gene expression could be harmful for achieving functional parameter sets of ion channel expression during developmental maturation or during pathological perturbations. Neurons are thought to target a certain desired set point in the high-dimensional 
parameter space of expression levels of ion channels. Our simulations show that the subspace 382 around these target values tends to be more densely filled with functional model parameters 383 than non-valid parameters (Figure 2C). Accordingly, despite fluctuations, high-dimensional 384 models are more likely to end up in functional subspaces. Even without the implementation of 385 homeostatic regulation processes, the chance of obtaining a functional ion channel expression level is relatively high. This puts random fluctuations in the expression of ion channels among the biologically plausible strategies to implement robust excitability profiles in neurons. Our computational analysis indicates that a complex high-dimensional parameter space supports the stability of neuronal excitability against perturbations that would push neurons into non-functional subspaces. The reason is that the topology of the high-dimensional space increases the likelihood of neurons returning into functional subspaces by random ion channel parameter adjustments.

Thus, due to the diversity of electrophysiological mechanisms, the cell is able to generate valid electrophysiological activity by random selection of parameters with a high chance of success despite stochastic fluctuations in the channel-coding genes. We showed that there was a clear relation between the number of intrinsic mechanisms and the chance to obtain a valid set of parameters from a random sample around a functional set point (Figures $2 \mathbf{B}, \mathbf{C}$, and 3). Furthermore, we showed that many other parameter combinations existed around a functional point in the parameter space that fulfilled our criteria for functional activity. While in a random $0-2 \times$ fold sample of the initial model, about $\sim 5.7 \%$ of the parameter combinations showed a valid GC activity, this proportion decreased steadily to $\sim 0.7 \%$ with a reduction of the model (Figure $2 \mathbf{B}$ ). In the closer surrounding of the baseline models this difference was even more obvious. While in the unreduced model in the close neighbourhood of $\pm 20 \%$ of the initial parameter sets over $80 \%$ of the models showed characteristic GC activity, in the heavily reduced model it was only about 30\% (Figure 2C).

Similar to (Olypher and Calabrese, 2007; Achard and De Schutter, 2006) we showed that near 407 each functional point in the parameter space many other parameter sets exist whose activity 408 match the activity of the original parameter set (Figure S6- S8). Instead of talking about 409 parameter sets, one should rather speak about subspaces that show functional behaviour. 410 These subspaces can have different densities of parameter sets showing characteristic elec- ${ }_{411}$ trophysiological activity. This depends to a great extent on the diversity of the channels 412 (Figures 2B, C, and 3A, Left panel). Furthermore, different valid subspaces with the same ${ }_{413}$ 
diversity differ in their density of functional solutions located in this subspace. In order to 414 be as robust as possible against perturbations and to simplify the process of parameter fit, it 415 seems reasonable for a neuron to target an as densely populated subspace as possible.

It is interesting to look at our random walk simulations (Figure 2D) in the context of evolution, 417 which can be interpreted as a random walk through the high-dimensional parameter space. 418 The path length between two valid points in the space on average requires macro-mutations 419 (larger step) in the 5 channels case whereas small gradual mutations can lead to the same 420 outcome in the 15 channel case. This indicates that, with a sufficient number of channels, a 421 valid spiking phenotype can be achieved in small evolutionary steps with high stability. Our 422 findings are in a strong agreement with the counter-intuitive hypothesis that degeneracy not ${ }_{423}$ only contributes to the robustness but also to the evolvability of biological systems (Whitacre ${ }_{424}^{42}$ and Bender, 2010).

\section{Conclusions and outlook}

We have put forward the law of large numbers as a possible explanation for our observations in the GC model. As a consequence of the law of large numbers, a model containing more ion 427 channels tends to exhibit a behaviour that is closer to its expected target behaviour (Figure 2). Accordingly, we were able to recover the amount of robustness observed in our full model when adding artificial ion channel isoforms (Figure 3). This is a strong indicator that indeed the number of ion channels and not their specific composition leads to the effect that we observed.

Future studies investigating the correlations between ion channel coding gene expressions within populations of GCs might be able to validate the results from our populations of GC models and the corresponding correlations between different conductances (Figure S4, Red squares). One of the assumptions here is that genetic expression of ion channels in neurons targets functional set points in the parameter space. In that particular case, the diversity of electrophysiological mechanisms seems to increase the chance of reaching a functional subspace around the target parameter, despite random fluctuations in gene expression.

Overall, our results suggest that the diversity of ion channels allows for increased robustness 441 and higher flexibility of finding a solution in the complex parameter space of a neuron's 442 
Biophysical complexity supports robust function

Schneider et al.

excitability. It will be interesting to investigate whether our findings here translate to other bio- ${ }_{443}$ logically complex systems, in which case they will most likely affect our general understanding ${ }_{444}$ of how evolution deals with complex organisms.

\section{Acknowledgments}

This work was supported by BMBF (No. 01GQ1406 - Bernstein Award 2013 to HC, No. ${ }_{447}$ 031L0229 - HUMANEUROMOD to PJ) and by funds from the von Behring Röntgen Founda- 448 tion to PJ. JT acknowledges support by the Johanna Quandt foundation. MS was supported ${ }_{449}$ by the International Max Planck Research School (IMPRS) for Neural Circuits in Frankfurt. 450 The authors declare to have no competing financial interests.

\section{Author contributions}

M.S., A.G., J.T., P.J., and H.C. designed the study. M.S. performed the simulations and analysed the data. M.S., A.G. J.T., P.J., and H.C. wrote the paper.

\section{Material and methods}

All simulations were performed in Matlab $2017 b$ (Mathworks, Natick, MA, USA). Single neuron ${ }_{456}$ simulations were performed using T2N (Beining et al. 2017, Www.treestoolbox. org/T2N), 457 a Matlab interface between the open source package TREES toolbox (Cuntz et al., 2010, 2011, 458 Www.treestoolbox.org) and the NEURON simulation environment (Hines and Carnevale, 459 1997. www.neuron.yale.edu). Predefined functions from TREES toolbox, T2N as well as additional custom Matlab code were used to generate and analyse the models. All code will be made available upon publication. 


\section{The granule cell (GC) model}

The GC model used in this study has been fully described in (Beining et al., 2017). Briefly, 464 the model was designed to reproduce passive and active GC properties as determined by 465 voltage and current clamp experiments, dendritic patch recordings of bAPs, and intracellular ${ }_{466}$ calcium imaging. In order to reduce the number of parameters and to speed up simulations $\quad 467$ we simplified the morphology by deleting the artificially added axon. The loss of the axon ${ }_{468}$ was compensated by slight changes of the maximum conductances in the axon initial segment ${ }_{469}$ (AIS). Since the HCN channel in its original form had no influence on control GC activity, we 470 did not take it into account. The compartment-specific distributions of ion channels are shown 471 in Table S1. Detailed descriptions of the individual ion channels can be found in Beining et al. 472 (2017). We used a realistic three-dimensional granule cell morphology from Schmidt-Hieber ${ }_{473}$ et al. (2007).

\section{Stimulation protocols and cost function}

Instead of using a single optimal error function, we decided to adopt a strategy that allows to take into account several potentially important properties of GC activity. To get a first impression of the "goodness of a model", we compared the experimental (Mongiat et al. 2009) and the model spiking-properties following a 200 ms lasting current injection of 50 and $90 \mathrm{pA}$. The stimulation protocol was as follows: $50 \mathrm{~ms}$ prerun without stimulation, followed by $200 \mathrm{~ms}$ somatic current injection of 50 and $90 \mathrm{pA}$ completed with a $50 \mathrm{~ms}$ long period without current injection.

We extracted the following 9 spiking properties (Figures 1 $\mathbf{A}$ ) from the raw traces of current ${ }_{483}$ injections with 50 and $90 p A$ :

1. Numbers of Spike fired within $200 m s$ current clamp.

2. Latency of first spike after stimulus onset in $m s$.

3. The voltage threshold was defined as the voltage at which the change of membrane ${ }_{487}$ potential exceeded $15 \frac{\mathrm{mV}}{\mathrm{ms}}$.

4. Average amplitude of spikes. 
5. The fast after hyperpolarisation (fAHP) amplitude was calculated as the voltage dif- 490 ference between the spiking threshold and the minimum potential within $5 \mathrm{~ms}$ after a 491 spike.

6. Absolute value of fast after hyperpolarisation (fAHP) amplitude.

7. The action potential width was measured at half the height of the spike amplitude. $\quad{ }_{494}^{4}$

8. Time interval in $m s$ between the first and second spike during current clamp.

9. The adaptation index $A I$ was calculated in the following manner: $A I=1-\frac{I S I_{1}}{I S I_{\text {end }}}$

The spiking features for any given parameter combination in the model were then compared with the same experimentally derived spiking features (Mongiat et al., 2009) and expressed in units of standard deviation. This approach allowed us to take into account the intrinsic variability of each feature separately. The overall fitness $F_{i}$ of spike feature $i$ was defined as: variability of each feature separately.

$$
F_{i}=\frac{\left|S F_{i}-\overline{S F}_{i, e x p}\right|}{S D_{i, e x p}}
$$

where $\overline{S F}_{i, \exp }$ refers to the average value of the spike feature $i$ and $S D_{i, \exp }$ to the standard ${ }_{501}$ deviation of the spike feature $i$ across all recorded GCs. The value of the spike feature of ${ }_{502}$ the corresponding model for a given parameter combination was $S F_{i}$. For a parameter ${ }_{503}$ combination to be accepted as a valid combination, it was required to fulfil the following 504 condition:

$$
P=\max \left(\frac{\left|S F_{i}-\overline{S F}_{i, e x p}\right|}{S D_{i, e x p}}\right)<2, \text { for } i=1,2, \ldots, 9
$$

The value of the Pareto efficiency $P$ corresponded to the fitness $F_{i}$ of the spiking feature $S F_{i} \quad{ }^{506}$ that deviated most from the experimental average. 


\section{The search algorithm}

To search for parameter sets that match our criteria for valid GC activity we combined random sampling with a gradient descent algorithm. In order to search for local minima we used a conjugate gradient descent technique (Press et al., 2007). Conjugate gradient descent techniques involve successive calculations of local gradients followed by the exploration of the parameter space along a vector derived from that gradient. Starting from a random or given point in the parameter space, we calculated the gradients for each dimension with two sample points to smooth the slopes. The algorithm evaluates the calculated gradients of the fitness function in each dimension and moves in the direction of the steepest descent with respect to the cost function. The sample points where calculated in steps of $\pm 5 \%$ of the corresponding parameter value. This procedure was then repeated until the method converged to a local minimum of the corresponding Pareto efficiency $P$. The successive line minimisation was done in conjugated directions, so that the successive minimisations were as independent as possible. Theoretically, this ensured that the parameter search found a local minimum of the target function $P$. For some initial parameter combinations, large areas of the parameter space were completely flat (i.e. the gradient was zero). This was especially the case when the initial models showed no spiking activity (Figure $\mathbf{S 2} \mathbf{B}$ ). In this case, we increased the size of the iteration steps consecutively by $\pm 5 \%$. If still (after increasing the step size to $\pm 50 \%$ ) no gradients other than zero were found or the local minima did not fulfil the criteria of functional GC excitability, we randomised the parameters in the next step in an iteratively increasing range (from $\pm 10 \%$ of the corresponding parameter values in steps of $\pm 10 \%$ up to $\pm 50 \%)$. The gradient descent algorithm was used to find the parameter settings of the reduced models. Starting from the full model (Figure 1 $\mathbf{C}$, Table S1, S2 and S3), we gradually reduced the number of ion channels, starting with the channels that influenced the cost function the least.

\section{Hyperplanes}

To learn more about the relationship of the set of valid models, we created linear combinations 534 of our best solutions. This method was adopted from Achard and De Schutter (2006) and 535 allowed us to better estimate whether the solutions lie on a common low-dimensional manifold 
within the high-dimensional parameter space of the GC model variants (Figure $\mathbf{S 6}-\mathbf{S 8}$ ). As 537 a first step, we created linear combinations out of weighted sums of a pair of solutions. We 538 weighted the parameters of the respective model between 0.1 and 0.9 with a step size of 0.1 . 539 The weighting of the second solution was chosen such that the sum of the weights was equal 540 to 1 . As soon as the Pareto efficiency of all evaluated linear combinations fulfilled the criteria 541 for characteristic GC spiking, we assumed that the respective models were connected. In the 542 next step, we created linear combinations of three different valid solutions to visualise the 543 hyperplanes in two dimensions. We used several triplets of valid parameter sets and weighted 544 two of them with values between -1.5 and 2.5 using a step size of 0.04 . The corresponding 545 grid of combinations was visualised in a two-dimensional plot. The weighting of the third 546 selected parameter set was chosen in a way that the sum of all weights was equal to 1 . The 547 hyperplanes consisted of several thousand points, whereby the parameter sets with negative 548 values were removed. As a result, each hyperplane had different boundaries and thus a 549 different size. Finally, for each of these points we ran simulations and calculated their Pareto 550 efficiency. The Pareto efficiency of the models without spiking behaviour was set to 6, which 551 explains the abrupt change of colour on the right side of Figure $\mathbf{S 6}$. The colour selection of the 552 plots allowed a clear distinction between the valid (green) and the nonvalid (blue) models. $\quad 553$

\section{Diversity expansion}

In order to generate models with controllable amounts of ion channels we used the reduced 5 -channel model as a basis. We then produced multiple instances each of the remaining

\section{Toy model}

We created a toy model to test whether the law of large numbers is a reasonable explanation for 563 the phenomena we observed in the GC model. In order to mimic the distribution of functional 564 
overlapping ion channel expressions in a population of GC models around a genetically 565 targeted functional set point we used randomly sampled variables between zero and two 566 (Figure 2E). A valid toy model is defined as having a smaller average deviation from the mean ${ }_{567}$ (targeted value) than 0.015 . By decreasing the sample range around the mean in steps of 0.1568 down to a sample range between 0.9 and 1.1 we change the intensity of fluctuations around 569 the target point (Figure 2E). 570

\section{References}

Abbott LF, LeMasson G (1993) Analysis of neuron models with dynamically regulated 572 conductances. Neural Computation 5:823-842.

Achard P, De Schutter E (2006) Complex parameter landscape for a complex neuron model. 574 PLoS Computational Biology 2:e94.

Aizenman CD, Akerman CJ, Jensen KR, Cline HT (2003) Visually driven regulation of intrinsic 576 neuronal excitability improves stimulus detection in vivo. Neuron 39:831-842. 577

Alonso LM, Marder E (2019) Visualization of currents in neural models with similar behavior ${ }_{578}$ and different conductance densities. eLife 8:e42722. 579

Bahl A, Stemmler MB, Herz AV, Roth A (2012) Automated optimization of a reduced layer 5580 pyramidal cell model based on experimental data. Journal of Neuroscience Methods 210:22-34. 581

Bean BP (2007) The action potential in mammalian central neurons. Nature Reviews Neuro- 582 science 8:451-465.

Beining M, Mongiat LA, Schwarzacher SW, Cuntz H, Jedlicka P (2017) T2N as a new tool ${ }^{584}$ for robust electrophysiological modeling demonstrated for mature and adult-born dentate 585 granule cells. eLife 6:e26517.

Britton OJ, Bueno-Orovio A, Van Ammel K, Lu HR, Towart R, Gallacher DJ, Rodriguez B ${ }_{587}$ (2013) Experimentally calibrated population of models predicts and explains intersubject 588 variability in cardiac cellular electrophysiology. PNAS 110:E2098-E2105. 
Coetzee WA, Amarillo Y, Chiu J, Chow A, Lau D, Mccormack T, Morena H, Nadal MS, Ozaita 590 A, Pountney D, Saganich M, Miera EVS, Rudy B (2006) Molecular diversity of K+ channels. 591 Annals of the New York Academy of Sciences 868:233-255.

Connors BW, Gutnick MJ (1990) Intrinsic firing patterns of diverse neocortical neurons. Trends 593 in Neurosciences 13:99-104.

Cuntz H, Bird AD, Beining M, Schneider M, Mediavilla L, Hoffmann FZ, Deller T, Jedlicka 595 P (2019) A general principle of dendritic constancy - a neuron's size and shape invariant 596 excitability. bioRxiv .

Cuntz H, Forstner F, Borst A, Häusser M (2010) One rule to grow them all: A general theory 598 of neuronal branching and its practical application. PLoS Computational Biology 6:e1000877. 599

Cuntz H, Forstner F, Borst A, Häusser M (2011) The TREES Toolbox-Probing the basis of 600 axonal and dendritic Branching. Neuroinformatics 9:91-96.

Drion G, O’Leary T, Marder E (2015) Ion channel degeneracy enables robust and tunable 602 neuronal firing rates. PNAS 112:E5361-E5370.

Druckmann S (2007) A novel multiple objective optimization framework for constraining 604 conductance-based neuron models by experimental data. Frontiers in Neuroscience 1:7-18. 605

Edelman GM, Gally JA (2001) Degeneracy and complexity in biological systems. 606 PNAS 98:13763-13768.

Elowitz MB, Levine AJ, Siggia ED, Swain PS (2002) Stochastic gene expression in a single cell. 608 Science 297:1183-1186.

Gal A, Eytan D, Wallach A, Sandler M, Schiller J, Marom S (2010) Dynamics of excitability over 610 extended timescales in cultured cortical neurons. Journal of Neuroscience 30:16332-16342. 611

Gjorgjieva J, Drion G, Marder E (2016) Computational implications of biophysical diversity 612 and multiple timescales in neurons and synapses for circuit performance. Current Opinion 613 in Neurobiology 37:44-52.

Goaillard JM, Marder E (2021) Ion channel degeneracy, variability, and covariation in neuron 615 and circuit resilience. Annual Review of Neuroscience Online ahead of print. 
Golowasch J, Marder E (1992) Ionic currents of the lateral pyloric neuron of the stomatogastric ${ }_{617}$ ganglion of the crab. Journal of Neurophysiology 67:318-331.

Golowasch J, Casey M, Abbott LF, Marder E (1999) Network stability from activity-dependent ${ }_{619}$ regulation of neuronal conductances. Neural Computation 11:1079-1096. 620

Golowasch J, Goldman MS, Abbott LF, Marder E (2002) Failure of averaging in the construction 621 of a conductance-based neuron model. Journal of Neurophysiology 87:1129-1131. 622

Gunay C, Edgerton JR, Jaeger D (2008) Channel density distributions explain spiking vari- ${ }_{623}$ ability in the globus pallidus: A combined physiology and computer simulation database 624 approach. Journal of Neuroscience 28:7476-7491.

Guo W, Jung WE, Marionneau C, Aimond F, Xu H, Yamada KA, Schwarz TL, Demolombe ${ }_{626}$ S, Nerbonne JM (2005) Targeted deletion of Kv4.2 eliminates Ito,f and results in electrical ${ }_{627}$ and molecular remodeling, with no evidence of ventricular hypertrophy or myocardial 628 dysfunction. Circulation Research 97:1342-1350. ${ }_{629}$

Hay E, Hill S, Schürmann F, Markram H, Segev I (2011) Models of neocortical layer 5b 630 pyramidal cells capturing a wide range of dendritic and perisomatic active properties. PLoS ${ }_{631}$ Computational Biology 7:e1002107.

Herrera-Valdez MA, McKiernan EC, Berger SD, Ryglewski S, Duch C, Crook S (2013) Relating 633 ion channel expression, bifurcation structure, and diverse firing patterns in a model of an 634 identified motor neuron. Journal of Computational Neuroscience 34:211-229.

Hille B (2001) Ion channels of excitable membranes Oxford University Press.

Hines ML, Carnevale NT (1997) The NEURON simulation environment. Neural Computa- 637 tion 9:1179-1209.

Jarsky T, Roxin A, Kath WL, Spruston N (2005) Conditional dendritic spike propagation 639 following distal synaptic activation of hippocampal CA1 pyramidal neurons. Nature Neuro- 640 science 8:1667-1676.

Keren N, Peled N, Korngreen A (2005) Constraining compartmental models using multiple 642 voltage recordings and genetic algorithms. Journal of Neurophysiology 94:3730-3742. 
Kirkpatrick S, Gelatt CD, Vecchi MP (1983) Optimization by simulated annealing. Sci- 644 ence 220:671-680.

Liu Z, Golowasch J, Marder E, Abbott LF (1998) A model neuron with activity-dependent ${ }_{646}$ conductances regulated by multiple calcium sensors. Journal of Neuroscience 18:2309-2320. 647

MacLean JN, Zhang Y, Goeritz ML, Casey R, Oliva R, Guckenheimer J, Harris-Warrick RM 648 (2005) Activity-independent coregulation of IA and Ih in rhythmically active neurons. 649 Journal of Neurophysiology 94:3601-3617.

MacLean JN, Zhang Y, Johnson BR, Harris-Warrick RM (2003) Activity-independent home- 651 ostasis in rhythmically active neurons. Neuron 37:109-120.

Marder E (2011) Variability, compensation, and modulation in neurons and circuits. 653 PNAS 108:15542-15548.

Marder E, Goaillard JM (2006) Variability, compensation and homeostasis in neuron and network function. Nature Reviews Neuroscience 7:563-574.

Marder E, O'Leary T, Shruti S (2014) Neuromodulation of circuits with variable parame- 657 ters: single neurons and small circuits reveal principles of state-dependent and robust 658 neuromodulation. Annual Review of Neuroscience 37:329-346.

Mateos-Aparicio P, Murphy R, Storm JF (2014) Complementary functions of SK and Kv7/M 660 potassium channels in excitability control and synaptic integration in rat hippocampal 661 dentate granule cells. Journal of Physiology 592:669-693.

Mishra P, Narayanan R (2021) Ion-channel regulation of response decorrelation in a heteroge- 663 neous multi-scale model of the dentate gyrus. Current Research in Neurobiology 2:100007. $\quad 664$

Mitchell M (1998) An Introduction to Genetic Algorithms MIT Press.

Mongiat LA, Espósito MS, Lombardi G, Schinder AF (2009) Reliable activation of immature 666 neurons in the adult hippocampus. PLOS ONE 4.

Nerbonne JM, Gerber BR, Norris A, Burkhalter A (2008) Electrical remodelling maintains 668 firing properties in cortical pyramidal neurons lacking KCND2-encoded A-type K+ currents. ${ }_{669}$ Journal of Physiology 586:1565-1579. 
O'Leary T, van Rossum MC, Wyllie DJ (2010) Homeostasis of intrinsic excitability in hip- 671 pocampal neurones: Dynamics and mechanism of the response to chronic depolarization. 672 Journal of Physiology 588:157-170.

O'Leary T, Williams AH, Caplan JS, Marder E (2013) Correlations in ion channel expression 674 emerge from homeostatic tuning rules. PNAS 110:E2645-E2654. 675

O’Leary T, Williams AH, Franci A, Marder E (2014) Cell types, network homeostasis, and 676 pathological compensation from a biologically plausible ion channel expression model. 677 Neuron 82:809-821.

Olypher AV, Calabrese RL (2007) Using constraints on neuronal activity to reveal compen- 679 satory changes in neuronal parameters. Journal of Neurophysiology 98:3749-3758. 680

Press WH, Teukolsky SA, Vetterling WT, Flannery BP (2007) Numerical Recipes Cambridge ${ }_{681}$ University Press.

Prinz AA, Billimoria CP, Marder E, A A, Alternative EM (2003) Analysis of databases of model 683 neurons. Journal of Neurophysiology pp. $3998-4015$.

Prinz AA, Bucher D, Marder E (2004) Similar network activity from disparate circuit parame- 685 ters. Nature Neuroscience 7:1345-1352.

Raj A, van Oudenaarden A (2008) Nature, nurture, or chance: stochastic gene expression and ${ }_{687}$ its consequences. Cell 135:216-226.

Rathour RK, Narayanan R (2019) Degeneracy in hippocampal physiology and plasticity. 689 Hippocampus 29:980-1022.

Rudy B (1988) Diversity and ubiquity of K channels. Neuroscience 25:729-749.

Schmidt-Hieber C, Jonas P, Bischofberger J (2007) Subthreshold dendritic signal processing and coincidence detection in dentate gyrus granule cells. Journal of Neuroscience 27:8430-8441.

Schulz DJ, Goaillard JM, Marder E (2007) Quantitative expression profiling of identified ${ }^{694}$ neurons reveals cell-specific constraints on highly variable levels of gene expression. 695 PNAS 104:13187-13191.

Schulz DJ, Goaillard JM, Marder E (2006) Variable channel expression in identified single and electrically coupled neurons in different animals. Nature Neuroscience 9:356-362. 
Sekulic V, Lawrence JJ, Skinner FK (2014) Using multi-compartment ensemble modeling as an 699 investigative tool of spatially distributed biophysical balances: Application to hippocampal 700 oriens-lacunosum/moleculare (O-LM) cells. PLOS ONE 9.

Sigal A, Milo R, Cohen A, Geva-Zatorsky N, Klein Y, Liron Y, Rosenfeld N, Danon T, Perzov N, 702 Alon U (2006) Variability and memory of protein levels in human cells. Nature 444:643-646. 703

Stegen M, Kirchheim F, Hanuschkin A, Staszewski O, Veh RW, Wolfart J (2012) Adaptive 704 intrinsic plasticity in human dentate gyrus granule cells during temporal lobe epilepsy. 705 Cerebral Cortex 22:2087-2101.

Swensen AM (2005) Robustness of Burst Firing in Dissociated Purkinje Neurons with Acute 707 or Long-Term Reductions in Sodium Conductance. Journal of Neuroscience 25:3509-3520. 708

Tononi G, Sporns O, Edelman GM (2002) Measures of degeneracy and redundancy in biologi- 709 cal networks. PNAS 96:3257-3262.

Turrigiano G, Desai NS, Rutherford LC (1999) Plasticity in the intrinsic excitability of cortical 711 pyramidal neurons. Nature Neuroscience 2:515-520.

Vanier MC, Bower JM (1999) A comparative survey of automated parameter-search methods 713 for compartmental neural models. Journal of Computational Neuroscience 7:149-171.

Whitacre J, Bender A (2010) Degeneracy: A design principle for achieving robustness and 715 evolvability. Journal of Theoretical Biology 263:143-153.

Young CC, Stegen M, Bernard R, Müller M, Bischofberger J, Veh RW, Haas CA, Wolfart J 717 (2009) Upregulation of inward rectifier K+ (Kir2) channels in dentate gyrus granule cells in 718 temporal lobe epilepsy. Journal of Physiology 587:4213-4233.

Zhang Y, Oliva R, Gisselmann G, Hatt H, Guckenheimer J, Harris-Warrick RM (2003) Over- 720 expression of a hyperpolarization-activated cation current (Ih) channel gene modifies the 721 firing activity of identified motor neurons in a small neural network. Journal of Neuro- 722 science 23:9059-9067. 


\section{Supporting information}

A
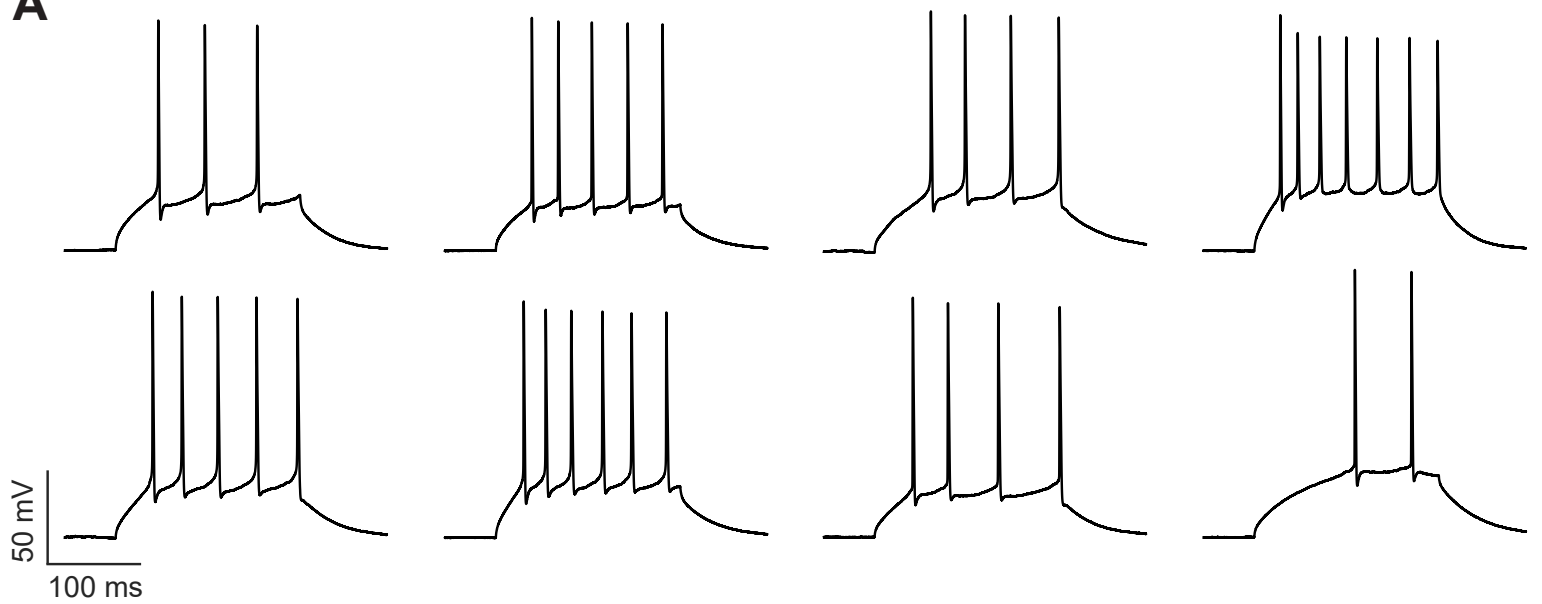

B

C
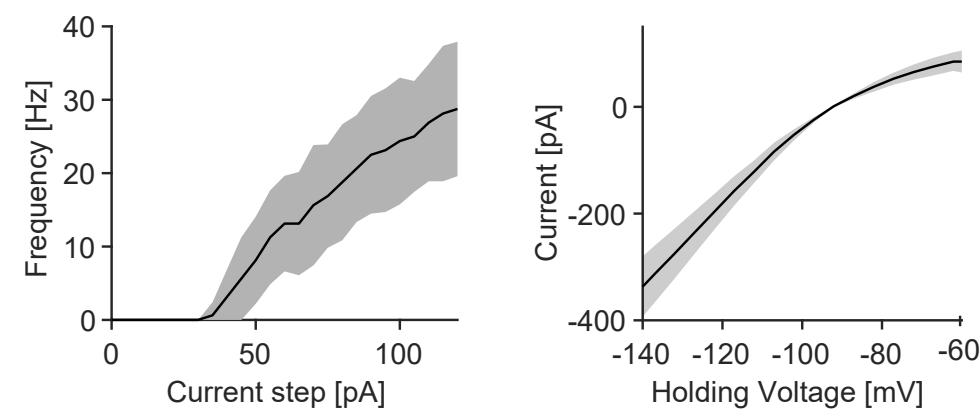

D

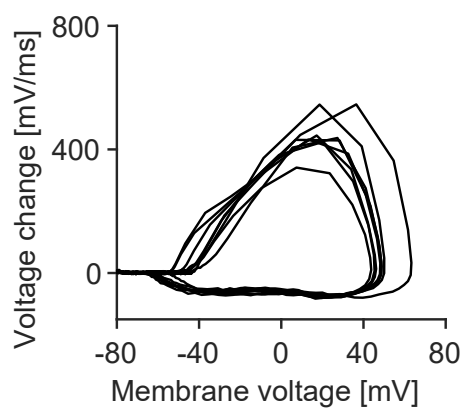

Figure S1. Electrophysiological properties of mouse GCs.

Experimental data from (Mongiat et al., 2009). A, Voltage traces of eight different GCs during $200 \mathrm{~ms}$ current clamp injection of $90 \mathrm{pA}$. B, Frequency of action potentials elicited by $200 \mathrm{~ms}$ lasting current injections (mean and standard deviation from raw traces, experimental standard deviation is shown as grey patches). C, Current-voltage (I-V) relationships (mean and standard deviation from raw traces, experimental standard deviation is shown as grey patches). $\mathbf{D}$, Phase plots of the first action potential during $90 p A$ current clamp. Modified from Figure 2 in Beining et al. (2017). 
A
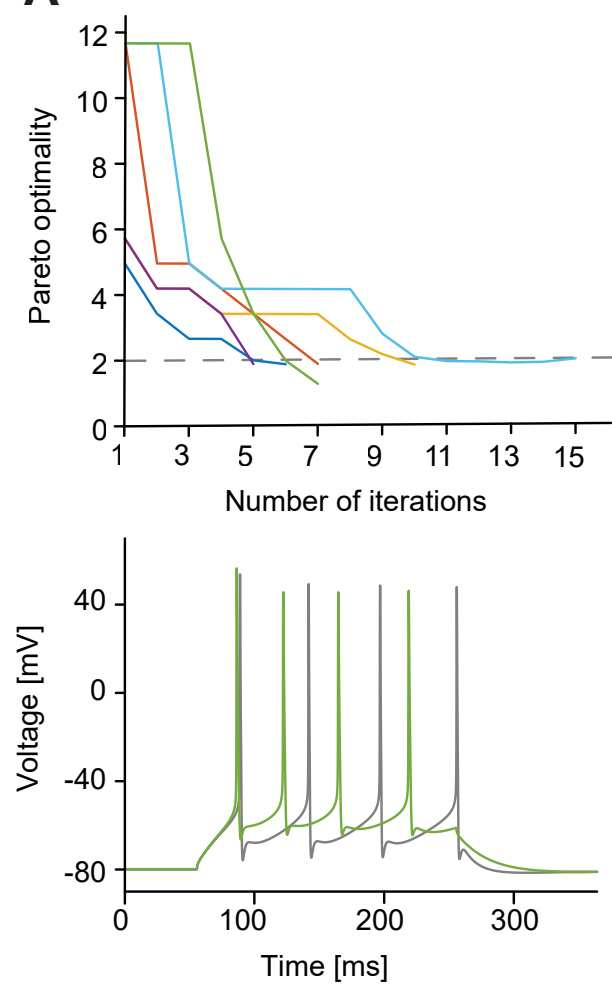

B
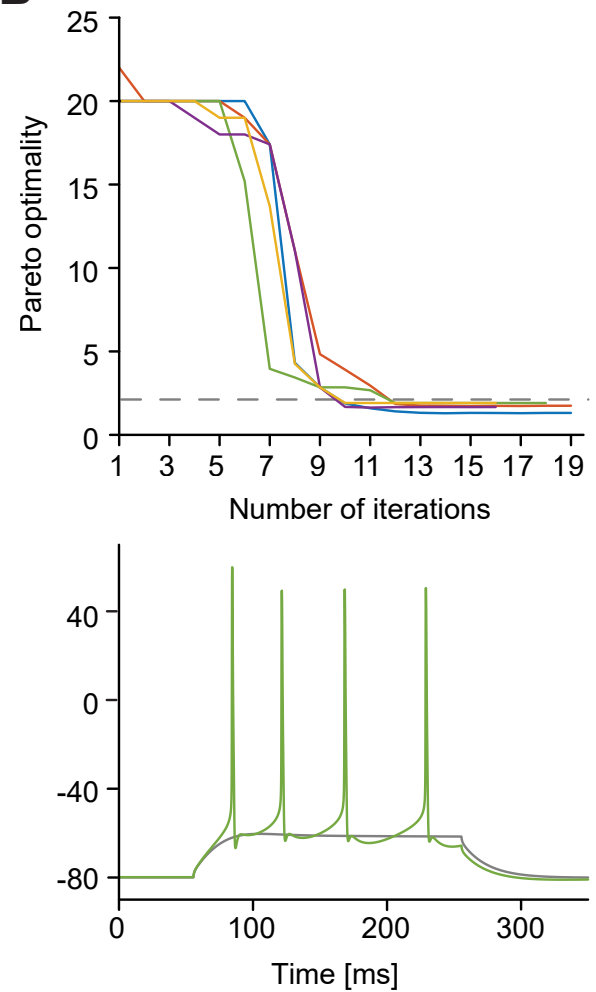

Figure S2. Gradient descent using multi-objective optimisation.

A, Temporal evolution of Pareto optimality (top) using the gradient descent method. Initial parameter combinations are random non-valid parameter combinations within a range between $0 \times$ and $2 \times$ the value in the reference parameter set. (bottom) Voltage traces of the model with initial parameter combinations (grey) and optimised parameters (green). B, Same as in A, but all initial parameter combinations were in a similar order of magnitude of Pareto optimality with corresponding models that did not even produce spikes. 
A

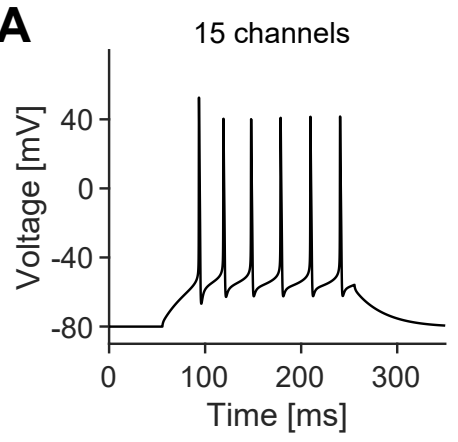

B

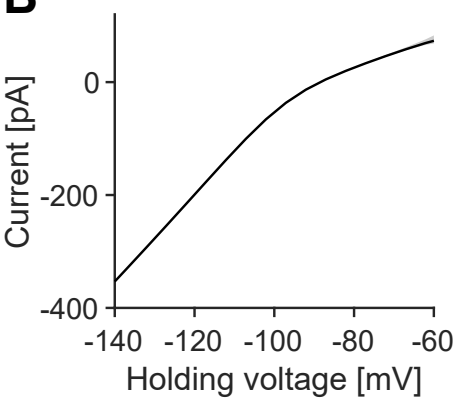

C
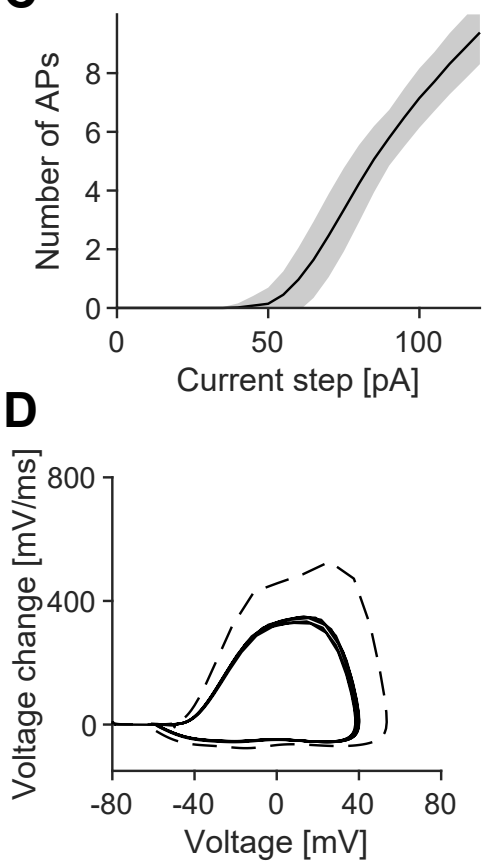

8 channels
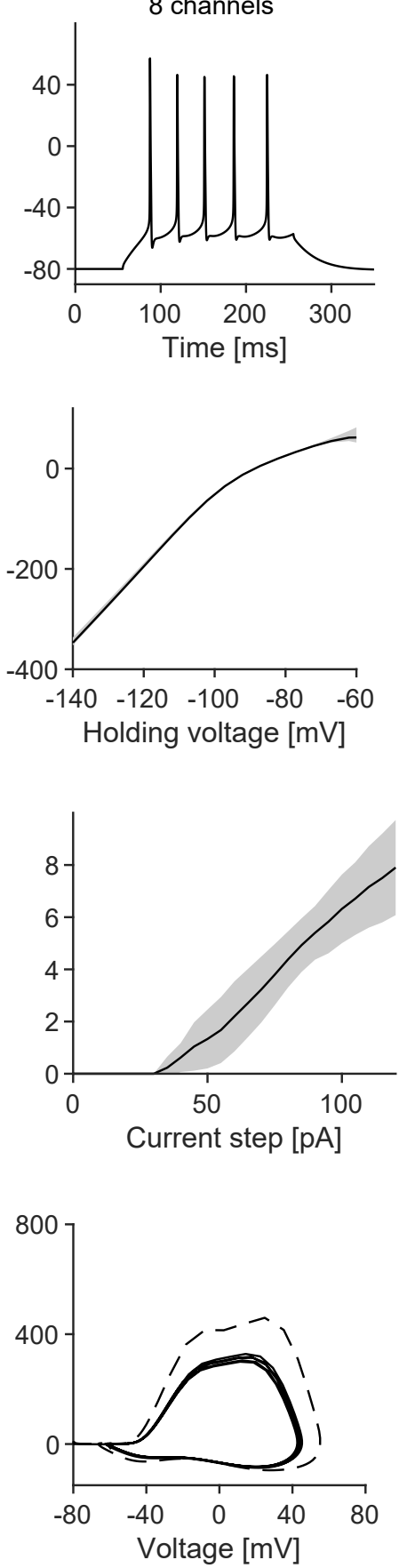

5 channels
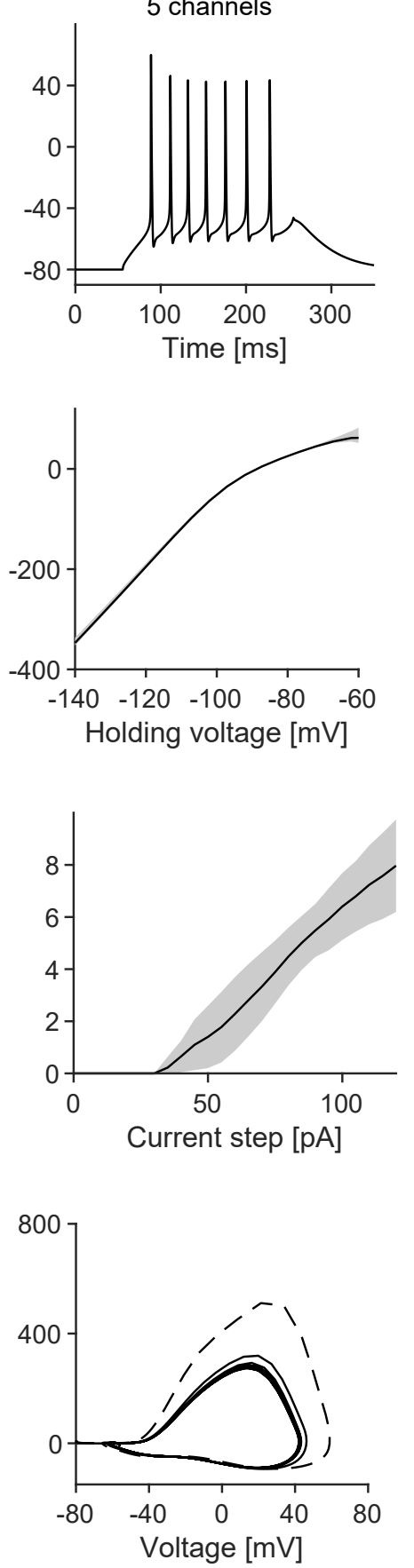

Figure S3. Comparison of the different GC models in Figure $1 \mathrm{C}$.

A-D, Similar panels as in Figure $\mathbf{S 1}$ for the different models and respective parameter combinations as in Figure 2 A. 
A

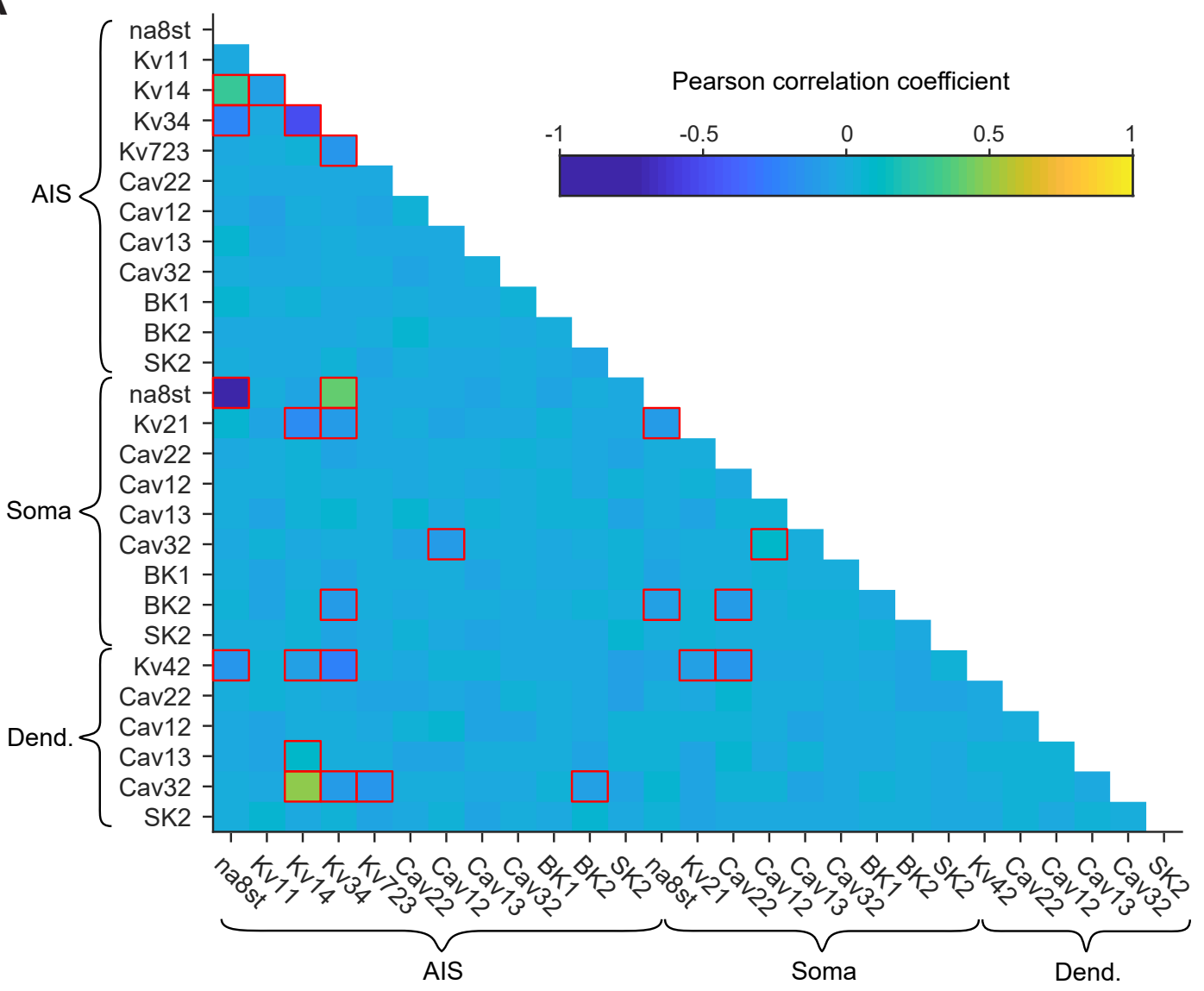

B

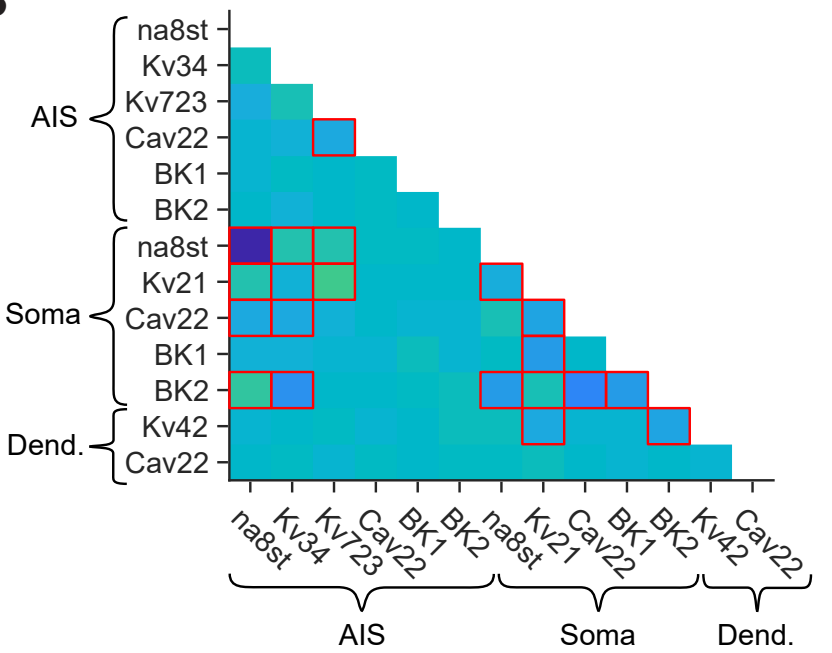

C

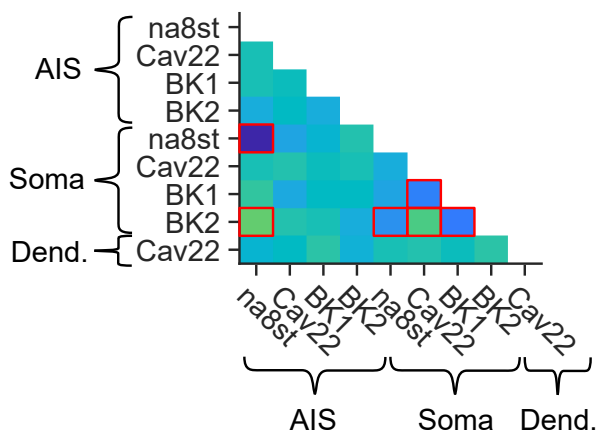

Figure S4. Correlations between pairs of channel conductances in the different populations.

Significant correlations are highlighted by red boxes ( $\mathrm{p}$-value $<0.01$ ). Pairwise correlations in population of A, 15-channel models, B, 9-channel models, C, 5-channel models. 

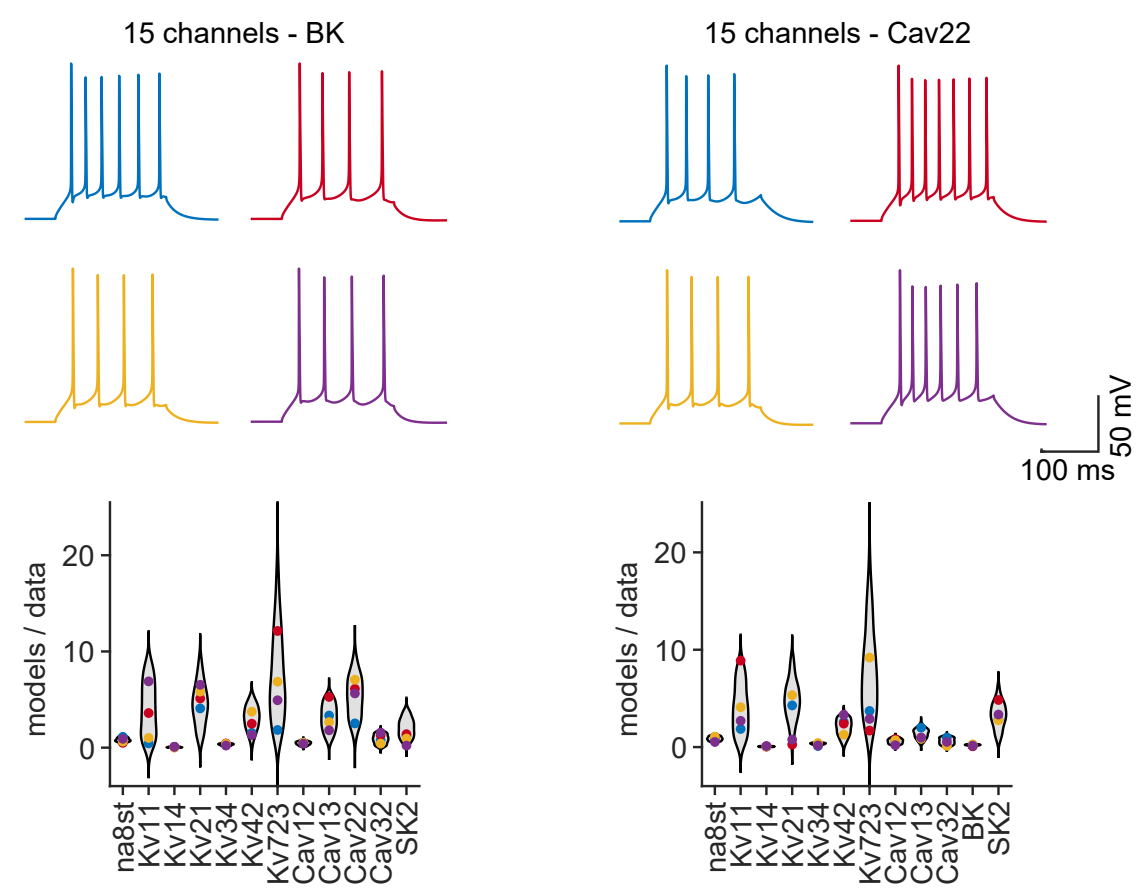

Figure S5. Valid parameter combinations in models that compensate for the knock-out of the BK (Left) and Cav22 (Right) channel.

Valid parameter combinations in the fully complex model are well spread and more stable as compared to reduced models. Activity traces of 4 randomly picked valid parameter combinations in models successfully compensating the corresponding knock-out (Top). Coloured dots illustrate conductance densities of the four valid parameter combinations shown in top traces (Bottom). Violin plots show the probability distribution of valid parameter combinations. Conductances are weighted by the surface area of the corresponding membrane regions. 
A

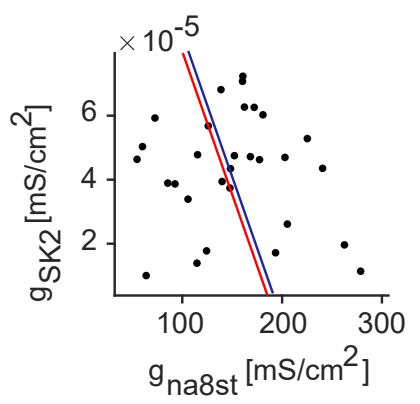

C

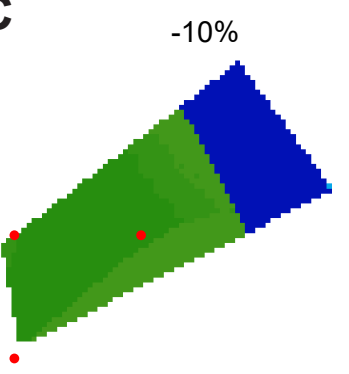

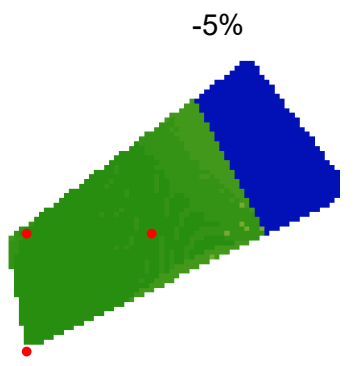

B
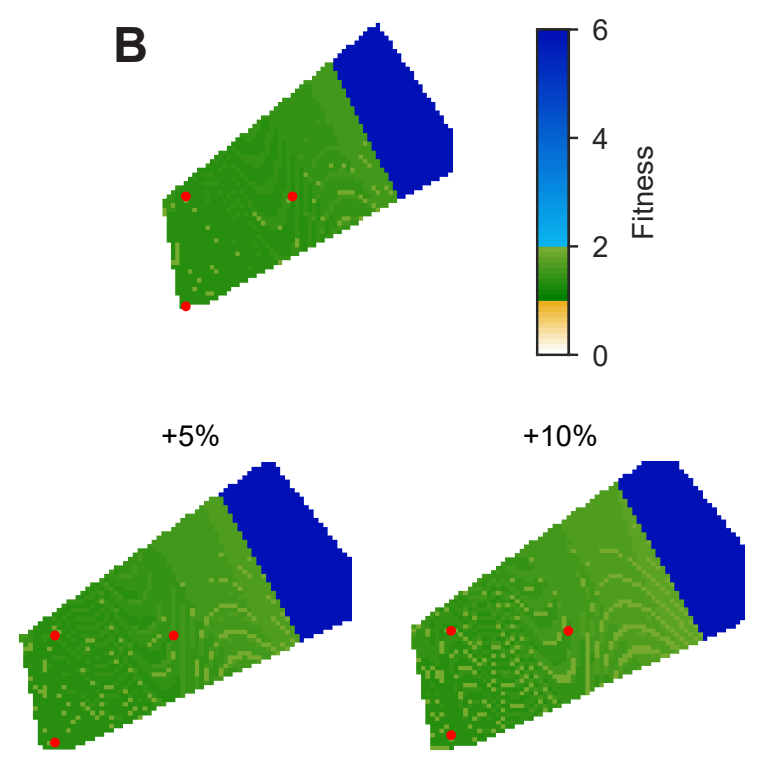

Figure S6. 2D illustrations of hyperplanes in the parameter space.

Hyperplane analysis inspired by Achard and De Schutter (2006) for the 15-channel model. A, The hyperplane of $\mathbf{B}$ is shown in red as projection onto $g_{N a 8 s t, A I S}$ vs. $g_{S K 2, A I S}$ plane. 25 randomly chosen valid parameter combinations are represented by dots. The blue hyperplane is parallel to the red and is defined by the addition of $10 \%$ of the SD of all solutions (in every dimension). B, Hyperplane defined by the three individuals on the red line in $\mathbf{A}$. The Fitness of all points is colour scaled. The three original individuals are highlighted as red dots. C, The red dots mark the places parallel to the 3 originally selected individuals. 
A

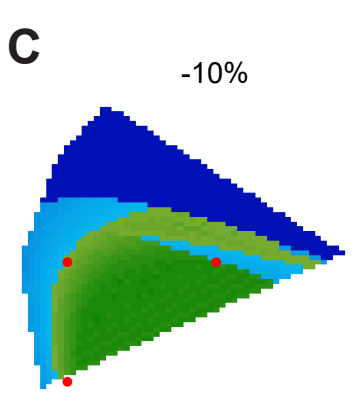

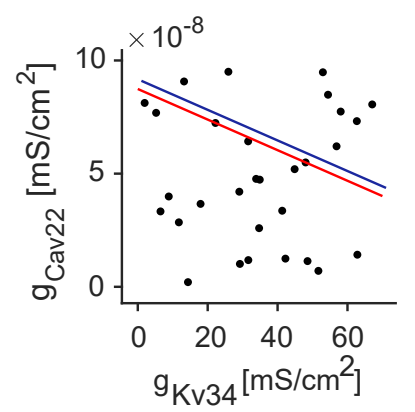

$-5 \%$

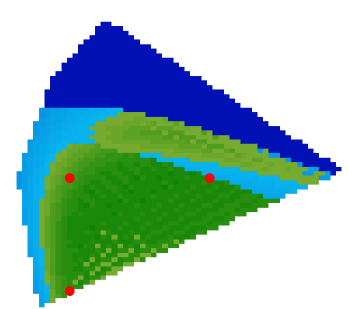

B
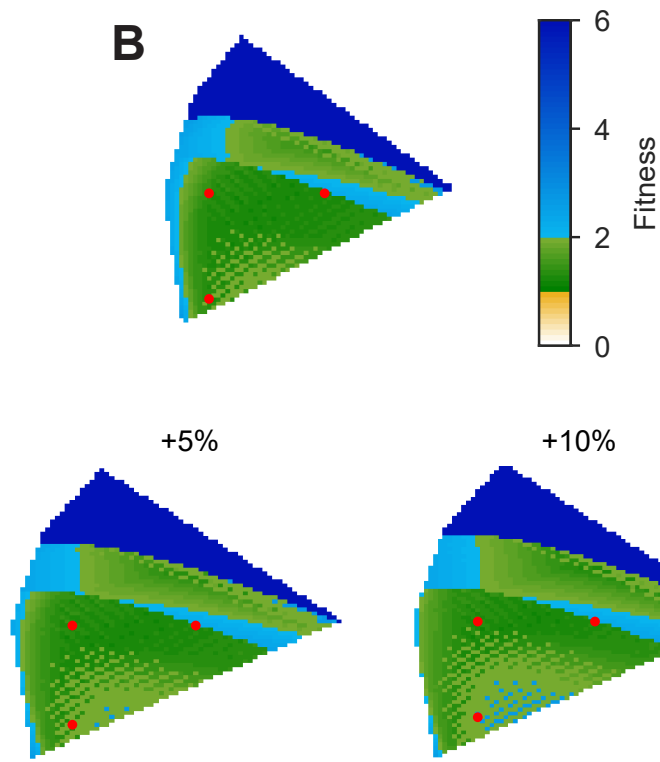

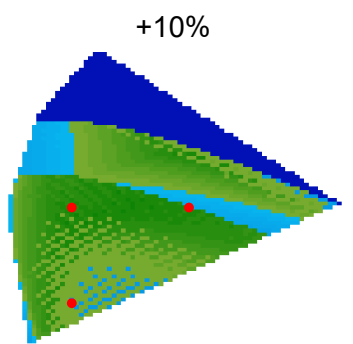

Figure S7. 2D illustrations of hyperplanes in the parameter space.

Hyperplane analysis inspired by Achard and De Schutter (2006) for the 9-channel model. A, The hyperplane of $\mathbf{B}$ is shown in red as projection onto $g_{K v 34, A I S}$ vs. $g_{C a v 22, A I S}$ plane. 25 randomly chosen valid parameter combinations are represented by dots. The blue hyperplane is parallel to the red and is defined by the addition of $10 \%$ of the SD of all solutions (in every dimension). B, Hyperplane defined by the three individuals on the red line in A. The Fitness of all points is colour scaled. The three original individuals are highlighted as red dots. $\mathbf{C}$, The red dots mark the places parallel to the 3 originally selected individuals. 

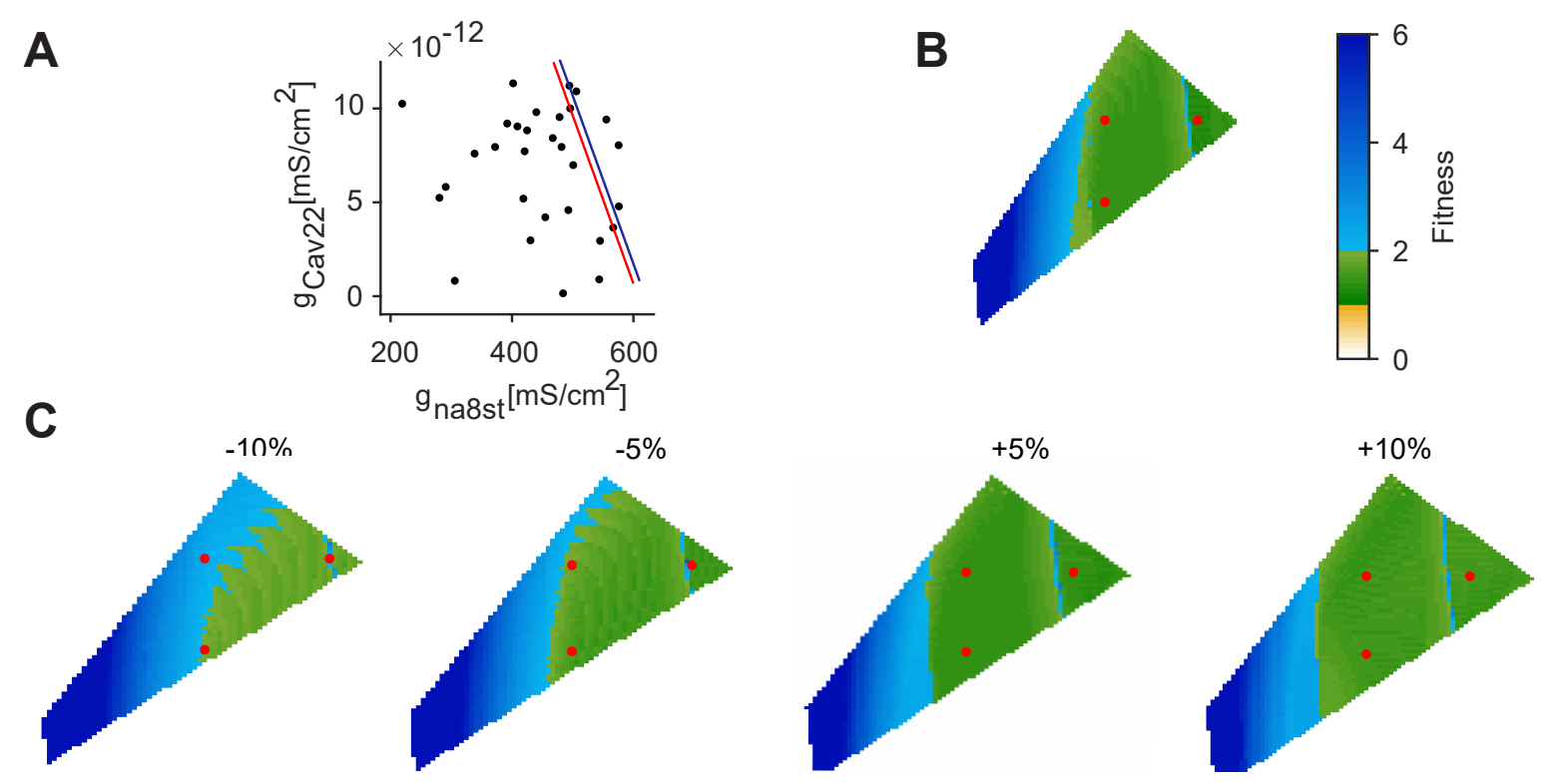

Figure S8. 2D illustrations of hyperplanes in the parameter space.

Hyperplane analysis inspired by Achard and De Schutter (2006) for the 5-channel model. A, The hyperplane of $\mathbf{B}$ is shown in red as projection onto $g_{n a 8 s t, A I S}$ vs. $g_{C a v 22, A I S}$ plane. 25 randomly chosen valid parameter combinations are represented by dots. The blue hyperplane is parallel to the red and is defined by the addition of $10 \%$ of the SD of all solutions (in every dimension). B, Hyperplane defined by the three individuals on the red line in A. The Fitness of all points is colour scaled. The three original individuals are highlighted as red dots. C, The red dots mark the places parallel to the 3 originally selected individuals. 
bioRxiv preprint doi: https://doi.org/10.1101/2021.05.04.442120; this version posted May 4, 2021. The copyright holder for this preprint (which

was not certified by peer review) is the author/funder, who has granted bioRxiv a license to display the preprint in perpetuity. It is made available under aCC-BY-NC-ND 4.0 International license.

Biophysical complexity supports robust function

Schneider et al.

\begin{tabular}{|c|c|c|c|}
\hline Name & AIS & Soma & Dendrite \\
\hline pas & $6.593 \times 10^{-6}$ & $1.385 \times 10^{-5}$ & $1.385 \times 10^{-5}$ \\
\hline Kir 2.1 & $6.741 \times 10^{-5}$ & $1.415 \times 10^{-4}$ & $1.415 \times 10^{-4}$ \\
\hline Na8st & 0.614 & 0.1478 & \\
\hline Kv 1.1 & $2.76 \times 10^{-4}$ & & \\
\hline Kv 1.4 & $1.77 \times 10^{-2}$ & & \\
\hline Kv 2.1 & & 0.0022 & \\
\hline Kv 3.4 & 0.6987 & & \\
\hline Kv 4.2 & & & 0.0039 \\
\hline $\mathrm{Kv} 7.2 / 3$ & 0.0031 & & \\
\hline Cav 1.2 & $3.1 \times 10^{-4}$ & $7.1 \times 10^{-5}$ & $2 \times 10^{-5}$ \\
\hline Cav 1.3 & $5.48 \times 10^{-6}$ & $2.5 \times 10^{-5}$ & $3.7 \times 10^{-6}$ \\
\hline Cav 2.2 & $3.19 \times 10^{-7}$ & $7.4 \times 10^{-5}$ & $5.8 \times 10^{-6}$ \\
\hline Cav 3.2 & $1.22 \times 10^{-5}$ & $1.6 \times 10^{-5}$ & $3.8 \times 10^{-5}$ \\
\hline \multicolumn{4}{|l|}{ BK } \\
\hline$\alpha$ & 0.0018 & $9.3 \times 10^{-4}$ & \\
\hline$\beta$ & 0.51 & 0.0148 & \\
\hline SK2 & $1.1 \times 10^{-5}$ & $3.7 \times 10^{-8}$ & $8.5 \times 10^{-7}$ \\
\hline
\end{tabular}

Table S1. Summary of ion channel densities and models implemented in the 15-channel model. Ion channels and their expression profiles in the corresponding morphological compartments. Conductance densities are given in units of $\mathrm{mS} / \mathrm{cm}^{2}$. 
Biophysical complexity supports robust function

Schneider et al.

\begin{tabular}{||cccc||}
\hline Name & AIS & Soma & Dendrite \\
\hline \hline pas & $6.593 \times 10^{-6}$ & $1.385 \times 10^{-5}$ & $1.385 \times 10^{-5}$ \\
\hline Kir 2.1 & $6.741 \times 10^{-5}$ & $1.415 \times 10^{-4}$ & $1.415 \times 10^{-4}$ \\
\hline Na8st & 0.4925 & 0.0881 & \\
\hline Kv 2.1 & & 0.0071 & \\
\hline Kv 3.4 & 0.0339 & & 0.0022 \\
\hline Kv 7.2/3 & 0.0074 & & \\
\hline Kv 4.2 & & & \\
\hline Cav 2.2 & $4.77 \times 10^{-11}$ & $4.5 \times 10^{-4}$ & \\
\hline BK & & 0.0043 & \\
$\alpha$ & $1.25 \times 10^{-7}$ & 0.0156 & \\
$\beta$ & 0.0148 & & \\
\hline
\end{tabular}

Table S2. Summary of ion channel densities and models implemented in the 9-channel model. Ion channels and their expression profiles in the corresponding morphological compartments. Conductance densities are given in units of $\mathrm{mS} / \mathrm{cm}^{2}$.

\begin{tabular}{||cccc||}
\hline Name & AIS & Soma & Dendrite \\
\hline \hline pas & $6.593 \times 10^{-6}$ & $1.385 \times 10^{-5}$ & $1.385 \times 10^{-5}$ \\
\hline Kir 2.1 & $6.741 \times 10^{-5}$ & $1.415 \times 10^{-4}$ & $1.415 \times 10^{-4}$ \\
\hline Na8st & 0.306 & 0.119 & \\
\hline Cav 2.2 & $5.82 \times 10^{-15}$ & $8.64 \times 10^{-4}$ & $1.22 \times 10^{-4}$ \\
\hline BK & & & \\
$\alpha$ & $1.16 \times 10^{-7}$ & 0.0132 & \\
$\beta$ & 1.321 & 0.0185 & \\
\hline
\end{tabular}

Table S3. Summary of ion channel densities and models implemented in the 5 -channel model. Ion channels and their expression profiles in the corresponding morphological compartments. Conductance densities are given in units of $\mathrm{mS} / \mathrm{cm}^{2}$. 